\title{
Impact of temperature and mode polarization on the acoustic phonon range in complex crystalline phases: A case study on intermetallic clathrates
}

\author{
S. R. Turner $\odot,{ }^{1,2,3}$ S. Pailhès $\odot,{ }^{3,{ }^{*}}$ F. Bourdarot $\odot,{ }^{4}$ J. Ollivier $\odot,{ }^{1}$ S. Raymond $\odot,{ }^{4}$ T. Keller $\odot,{ }^{5,6}$ Y. Sidis, ${ }^{7}$ J.-P. Castellan, ${ }^{7,8}$ \\ P.-F. Lory ${ }^{1},{ }^{1,2}$ H. Euchner $\odot,{ }^{9}$ M. Baitinger $\odot,{ }^{10}$ Yu. Grin, ${ }^{10}$ H. Schober, ${ }^{1}$ M. de Boissieu, ${ }^{2}$ and V. M. Giordano ${ }^{3}{ }^{3}$ \\ ${ }^{1}$ Institut Laue-Langevin, Grenoble F-38042 Grenoble cedex, France \\ ${ }^{2}$ Université Grenoble Alpes, CNRS, Grenoble-INP, SIMaP, F-38000 Grenoble, France \\ ${ }^{3}$ Institute of Light and Matter, UMR5306 Université Lyon 1-CNRS, Université de Lyon F-69622 Villeurbanne cedex, France \\ ${ }^{4}$ Université Grenoble Alpes, CEA, IRIG, MEM, MDN, F-38000 Grenoble cedex, France \\ ${ }^{5}$ Max-Planck-Institut für Festkörperforschung, Heisenbergstr. 1, D-70569 Stuttgart, Germany \\ ${ }^{6}$ Forschungsneutronenquelle Heinz Maier-Leibnitz, Technische Universität München, D-85748 Garching, Germany \\ ${ }^{7}$ Université Paris-Saclay, CNRS, CEA, Laboratoire Léon Brillouin, F-91191, Gif-sur-Yvette, France \\ ${ }^{8}$ Institut für Festkörperphysik, Karlsruher Institut für Technologie, D-76021 Karlsruhe, Germany \\ ${ }^{9}$ Helmholtz Institute Ulm for Electrochemical Energy Storage, Helmholtzstr. 11, D-89081 Ulm, Germany \\ ${ }^{10}$ Max-Planck-Institut für chemische Physik fester Stoffe, D-01187 Dresden, Germany
}

(Received 13 July 2020; accepted 25 November 2020; published 8 January 2021)

\begin{abstract}
The low and weakly temperature-varying lattice thermal conductivity, $\kappa_{L}(\mathrm{~T})$, in crystals with a complex unit cell such as type-I clathrates is assumed to originate from a reduced momentum and energy space available for propagative lattice vibrations, which is caused by the occurrence of low-energy optical phonon modes. In the context of ab initio self-consistent phonon (SCP) theory, it has been shown that the cubic and quartic anharmonic interactions result in a temperature-induced energy renormalization of these low-lying optical branches which contributes to the anomalous behavior of $\kappa_{L}(\mathrm{~T})$ in structurally ordered type-I clathrates [T. Tadano and $\mathrm{S}$. Tsuneyuki, Phys. Rev. Lett. 120, 105901 (2018)]. By means of inelastic neutron scattering, we provide evidence for this energy renormalization in temperature, which has been resolved for transversely and longitudinally polarized phonons in the single crystal type-I clathrate $\mathrm{Ba}_{7.81} \mathrm{Ge}_{40.67} \mathrm{Au}_{5.33}$. By mapping the neutron intensity in the momentum space, we demonstrate the coherent character of the low-lying optical phonons. The overall phonon spectrum and dynamical structure factors are satisfactorily reproduced by ab initio harmonic calculations using density functional theory with the meta-GGA SCAN functional and a fully ordered structure. However, a polarization-dependent cutoff energy with opposing temperature shifts for longitudinal and transverse acoustic dispersions is experimentally observed which is not reproduced by the simulations. Anharmonicity affects the energies of the low-lying optical phonons in the transverse polarization, which compares quantitatively well with available results from SCP theory, whereas differences are observed for the longitudinal polarization.
\end{abstract}

DOI: 10.1103/PhysRevResearch.3.013021

\section{INTRODUCTION}

Tailoring the lattice thermal conductivity, $\kappa_{L}$, of energyefficient semiconductors is a common materials issue in many applications such as for thermoelectric [1] and photovoltaic [2,3] conversion, phase change memories [4], and battery electrodes $[5,6]$. In the search for low $\kappa_{L}$, the main strategy is the use of "complexity" at multiple length scales, from structural complexity within the crystal unit cell, to disorder, short-range order, and nanostructuring [7-9]. Crystals with

\footnotetext{
*To whom correspondence should be addressed: stephane.pailhes@univ-lyon1.fr

Published by the American Physical Society under the terms of the Creative Commons Attribution 4.0 International license. Further distribution of this work must maintain attribution to the author(s) and the published article's title, journal citation, and DOI.
}

a high structural complexity and chemical bonding inhomogeneity [10], such as tetrahedrites [11] or type-I clathrates [12], often have a very low and almost temperature independent $\kappa_{L}$ of $\sim 0.5-2 \mathrm{Wm}^{-1} \mathrm{~K}^{-1}$ in the $50-500 \mathrm{~K}$ range. The current understanding is that the heat conduction is mostly conveyed by well-defined acoustic phonons, which exist only in a limited range of the energy and momentum phase space, delimited by a continuum of nondispersive optical phonon bands [13-18]. The onset of this continuum at low energy, labeled $E_{1}$, defines the upper energy limit of the acoustic regime such that it has been associated with a phononic low-pass acoustic filter [15] or a modified Debye energy [16,19]. $E_{1}$ can be changed by varying the chemical composition [13] or the structural topology [20]. Using the Boltzmann transport equation for phonons, the acoustic contribution of $\kappa_{L}^{a c}$ is given by a cumulative spectral integral $[13,15,19]$ :

$$
\kappa_{L}^{a c}(T)=\sum_{\nu} \int_{0}^{E_{1}^{\nu}(T)} \kappa_{L}^{a c, v}\left(\omega_{\mathbf{q}}\right) \rho^{\nu}\left(\omega_{\mathbf{q}}\right) d \omega,
$$


where $\kappa_{L}^{a c, v}(\omega)$ is the mode thermal conductivity, $\rho^{\nu}(\omega)$ is the density of states per mode (DOS), $v$ is the longitudinal/transverse polarization index and $\omega_{\mathbf{q}}$ stands for the phonon relation dispersions. In addition, any variation in $E_{1}$ changes the whole phonon-phonon scattering phase space, thus impacting the acoustic phonon lifetimes entering into $\kappa_{L}^{\nu}(\omega)[17,21]$. Explaining the combined relationship between the complex crystal structure and related defects, the acoustic phonon properties, and the nature of $E_{1}$, remains a fundamental challenge.

Type-I clathrates contain 46 framework atoms of mostly group 14 elements which arrange in a 3D covalent host network of face-sharing polyhedral cages that encapsulate alkali or alkali earth guest cations [12]. The structure is usually described using the cubic space group $\mathrm{P} m \overline{3} n$ (group 223) with a lattice parameter of about $1 \mathrm{~nm}$. The cutoff energy $E_{1}$ is defined as the center of a distribution of optical phonon modes related to the dynamics of guest atoms located at the $6 d$-Wyckoff sites in tetrakaidecahedral $\left(5^{12} 6^{2}\right)$ host cages. These modes lead to well-defined peaks in the phonon DOS $[15,17,18,21]$ which results in a large deviation from the Debye-like $T^{3}$ temperature dependence of the lattice specific heat $\left(C_{p}\right)$ at low temperature $[25,26]$. In literature, the temperature at the maximum of the $T^{3}$-normalized heat capacity is commonly referred to as an Einstein temperature and corresponds well to $E_{1}$ in the phonon spectrum which we recall as the upper limit of the integral in Eq. (1). Recently, a phenomenological universal relation has been revealed in type-I clathrates between $\kappa_{L}$ and the product of the average sound velocity and $E_{1}$ [19]. The nature of the low-lying guest optical phonons with energies around $E_{1}$ is particularly intriguing. These modes are characterized by a very low phonon participation ratio $(\lesssim 0.1)$, which is interpreted either as a signature of localization [27,28] or an effect of mode confinement $[13,14,17]$. In this latter case, the phonon is viewed as a Bloch state confined to a relatively small atomic pattern, the $\mathrm{Ba}(6 d)$ atoms in this case, within the large complex unit cell whose periodic repetitions result in a special character. Moreover, the flatness of their dispersion and the concomitant high DOS provides a large momentum- and energy-conserving phase space for three-phonon scattering processes involving the acoustic modes [17,21].

The $\mathrm{Ba}_{7.81} \mathrm{Ge}_{40.67} \mathrm{Au}_{5.33}$ structure [29-31], which will be dealt with in this paper, is shown in Fig. 1(a). Besides the structural complexity, the dative $\mathrm{Au}-\mathrm{Ba}$ bonding of the $\mathrm{Au}-$ substitution in $\mathrm{Ba}_{7.81} \mathrm{Ge}_{40.67} \mathrm{Au}_{5.33}$ results in an off-centering of $\mathrm{Ba}$ atoms in the tetrakaidecahedral cages $[13,32,33]$. No correlation among the off-centering sites has been observed experimentally, indicating that they can be viewed as point defects. In the type-I structure $\mathrm{Ba}_{8} \mathrm{Ge}_{40} \mathrm{Au}_{6}$, molecular dynamics simulations performed at $300 \mathrm{~K}$ on a $2 \times 2 \times 2$ supercell with independent random substitutions of $\mathrm{Au}$ atoms found no evidence for a correlated $\mathrm{Au} / \mathrm{Ge}$ defect structure, thus no ordering among the off-centered Ba positions (see the Supplementary Material in Ref. [13]).

All ab initio phonon and $\kappa_{L}$ calculations reported for type-I clathrates have been carried out on the fully ordered model structure, with centered $\mathrm{Ba}$ atoms and full site occupation of all sites. The experimental phonon spectrum in type-I clathrates is qualitatively reproduced quite well by harmonic
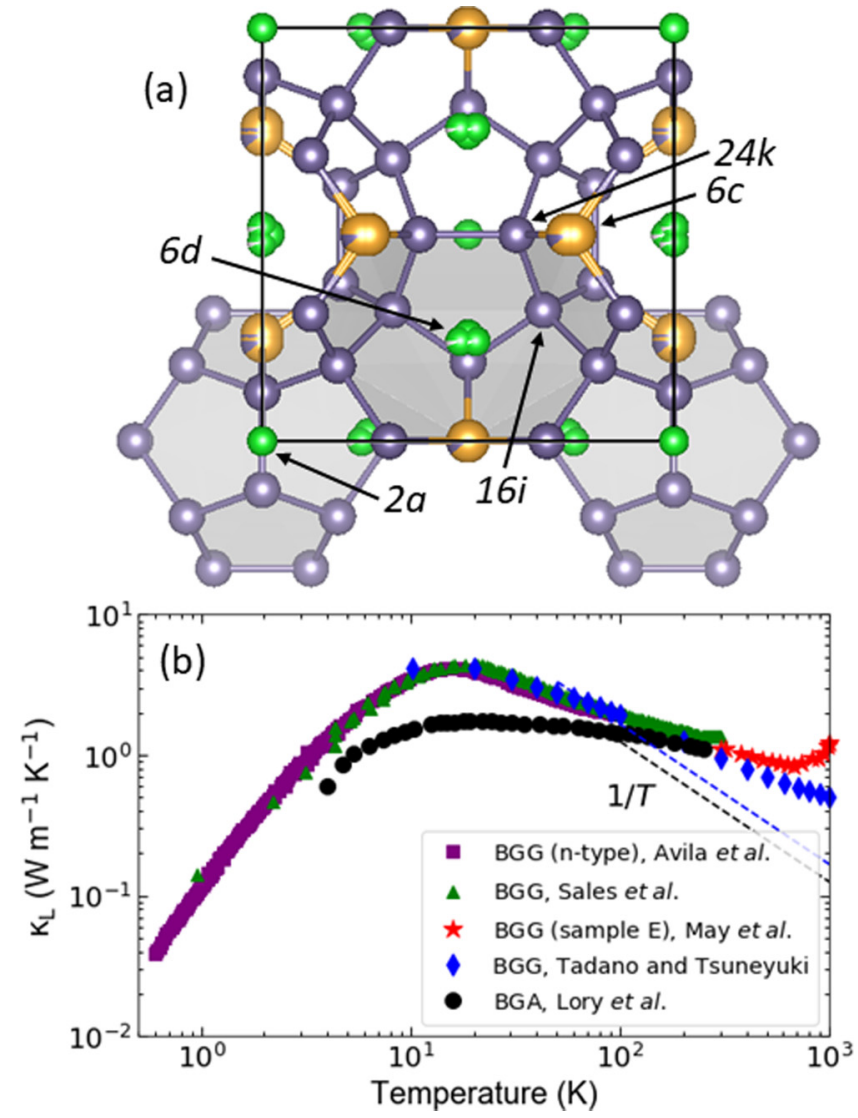

FIG. 1. (a) Crystallographic structure of the type-I clathrate $\mathrm{Ba}_{7.81} \mathrm{Ge}_{40.67} \mathrm{Au}_{5.33}$ (BGA) The cubic unit cell (space group $\mathrm{P} m \overline{3} n$ ) contains two tetrakaidecahedral $\left(5^{12} 6^{2}\right)$ and five dodecahedral $\left(5^{12}\right)$ host cages formed by Ge atoms (light gray) with guest Ba (green) atoms encapsulated inside. One to three $\mathrm{Au}$ atoms (gold) substitute $\mathrm{Ge}$ atoms at the Wyckoff site $6 c$, which results in a slight distortion of the tetrakaidecahedron and an off-centering of the Ba atoms inside [13]. (b) The lattice thermal conductivity, $\kappa_{L}$, for BGA (black circles) [13] is compared to different experimental measurements of $\kappa_{L}$ for $\mathrm{Ba}_{8} \mathrm{Ge}_{30} \mathrm{Ga}_{16}$ (BGG), including those by Avila et al. [22], Sales et al. [23], May et al. [24], and the theoretical calculations of Tadano and Tsuneyuki [43]. Black and blue dashed lines show the deviation of $\kappa_{L}$ from $1 / T$ for both BGA and BGG, respectively, at higher temperatures.

$a b$ initio calculations [13-15]. Quantitatively, ab initio lattice dynamics studies of Ge clathrates showed decreased acoustic and low-lying optical mode energies in comparison to the experimental data (up to $40 \%$ ), a discrepancy which has been recently overcome by the use of the meta-generalized-gradient approximation (meta-GGA) functional SCAN (strongly constrained and appropriately normed) for the exchange and correlation energy [34]. Ab initio simulations in perturbation theory, when limited to three-phonon processes, predicts a $1 / T$ temperature dependence for acoustic phonon lifetimes and $\kappa_{L}(T)$, which drastically fails to reproduce the experimental $\sim T^{-0.25}$ in $\mathrm{Ba}_{7.81} \mathrm{Ge}_{40.67} \mathrm{Au}_{5.33}$ [13] [see Fig. 1(b)], and also in other complex crystals like the $\sim T^{-0.1}$ dependence in the quasicrystal approximant $\mathrm{o}-\mathrm{Al}_{13} \mathrm{Co}_{4}$ [35]. For the latter case, molecular dynamics simulations on an ordered model structure yield a $T^{-0.5}$ dependence, whereas the inclusion 
of random disorder results in much closer agreement with experiment. This points not only to the importance of disorder, but also to either higher order anharmonicity or the effect of phonon energy renormalization including the polarization mixing of phonon eigenvectors, which are included in the molecular dynamics simulations but not in most ab initio based calculations.

A significant improvement to the $a b$ initio approach has been achieved by self-consistent phonon (SCP) theory, which nonperturbatively treats the effects of anharmonicity [36-42]. An SCP study on an ordered model of $\mathrm{Ba}_{8} \mathrm{Ge}_{30} \mathrm{Ga}_{16}$ reveals that the quartic anharmonicity leads to a softening of $E_{1}(T)$ upon cooling, surpassing the usual hardening effect due to thermal expansion. Although this softening accounts for less than $10 \%$ of $E_{1}(T)$ in the $0-300 \mathrm{~K}$ range, i.e., about $1 \mathrm{meV} \sim$ $12 \mathrm{~K}$, it leads to a closer $\kappa_{L}(T)$ matching [43]. This high sensitivity of $\kappa_{L}(T)$ to $E_{1}(T)$ results in the peculiar vibrational nature of the optical modes at $E_{1}(T)$. It should be mentioned here that the $\mathrm{Ba}_{8} \mathrm{Ge}_{30} \mathrm{Ga}_{16}$ SCP calculations assume that phonon polarization vectors are not affected by anharmonicity [43], which is a common approximation in complex crystals in order to limit the computational cost $[38,41]$.

In this paper, we provide substantial experimental evidence of the importance of the anharmonic processes (resulting from cubic and quartic terms) for the type-I clathrate $\mathrm{Ba}_{7.81} \mathrm{Ge}_{40.67} \mathrm{Au}_{5.33}$ by investigating the propagation direction, and the polarization and temperature dependencies of the cutoff energy $E_{1}^{v}(T)$ by inelastic neutron scattering on a high-quality single crystal. By probing the mode symmetry in momentum space at different energies, we show that the distribution of the neutron intensity related to the low-lying optical vibrations is structured in the momentum space within a Brillouin zone, and from one Brillouin zone to another. This distribution of intensity in momentum as well as the overall phonon energies are satisfactorily reproduced by ab initio harmonic calculations using the (meta-GGA) functional SCAN done on a fully ordered structure. We confirm the agreement between the experimental results and simulations for phonon dispersions obtained along different high-symmetry directions and with both transverse and longitudinal polarizations.

However, some differences remain, especially in the region of the phase space where acoustic and optical phonons are hybridized. Experimentally, a polarization dependence of the acoustic-optical phonon coupling is observed such that the origin and the value of $E_{1}$ in the longitudinal and transverse acoustic (LA, TA) polarizations are different and exhibit opposite temperature dependencies, the effect of optical mode hardening with increasing temperature being found only for the lowest mode in the transverse polarization. This difference, which is not observed in our ab initio harmonic simulations using a fully ordered model, indicates either an effect of the particular defect cage structure caused by the Au substitutions or a more subtle anharmonic effect involving phonon polarization.

Furthermore, and in light of the recent SCP method calculations on $\mathrm{Ba}_{8} \mathrm{Ge}_{30} \mathrm{Ga}_{16}$ [43], this experimental case allows us to quantitatively compare clathrate anharmonicity as found by both experimental and theoretical approaches. The rate of change in energy of $E_{1}(T)$ in $\mathrm{Ba}_{7.81} \mathrm{Ge}_{40.67} \mathrm{Au}_{5.33}$ was properly measured in the temperature range $100-550 \mathrm{~K}$. After
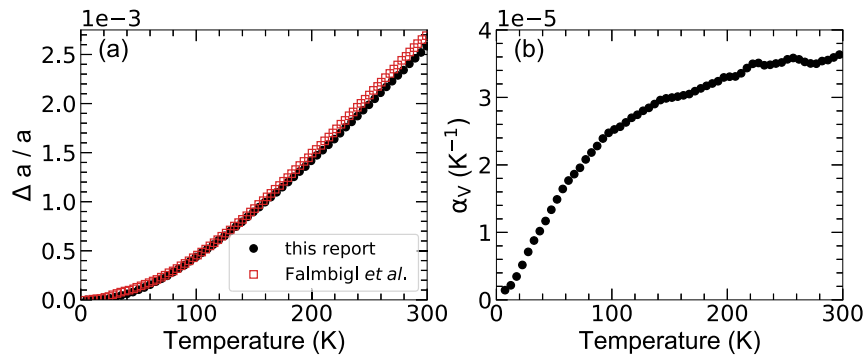

FIG. 2. (a) The rate of thermal expansion, $\Delta a / a$, for $\mathrm{Ba}_{7.81} \mathrm{Ge}_{40.67} \mathrm{Au}_{5.33}$ has been measured with Larmor diffraction on TRISP@FRM-II (black circles) and compared to that of Falmbigl et al. (empty red squares) [50]. The numerical derivative of $\Delta a / a$ is the linear thermal expansion coefficient, $\alpha_{L}$. The volumetric thermal expansion coefficient, $\alpha_{V}=3 \alpha_{L}$, is plotted in (b).

the subtraction of the thermal expansion contribution from this rate of change, the anharmonic contribution in the thermal shift of $E_{1}(T)$ has been experimentally determined and directly compared to that of the quartic and cubic terms of the SCP method calculations for $\mathrm{Ba}_{8} \mathrm{Ge}_{30} \mathrm{Ga}_{16}$, providing experimental validation of the SCP method calculations for determining anharmonic effects in clathrates.

\section{METHODS}

Inelastic neutron scattering (INS) measurements were performed on the same high-quality single crystal of the type-I clathrate $\mathrm{Ba}_{7.81} \mathrm{Ge}_{40.67} \mathrm{Au}_{5.33}$ as in our previous work [13], whose temperature dependence of the lattice thermal conductivity, $\kappa_{L}(T)$, is shown in Fig. 1(b). The structural study, the chemical disorder caused by Au substitutions, and the thermal characterizations were reported in Ref. [13]. In addition, we have precisely measured its lattice thermal expansion, as depicted in Fig. 2, by means of neutron Larmor diffraction on the triple-axis spectrometer TRISP at the Heinz MaierLeibnitz Zentrum (FRM-II, Germany) (see Appendix A 3). The INS intensity was recorded over a wide range of the momentum and energy phase space at 150,300 , and $530 \mathrm{~K}$ on the cold-neutron time-of-flight (TOF) spectrometer IN5 at the Institut Laue-Langevin (ILL, France). Details on the experimental settings, on the TOF instrumental resolution in momentum and in energy, and on the integration parameters used to produce the experimental phonon dispersions from fits of 1D-energy cuts at constant momentum (raw data and fits are shown in Appendix F), the high resolution Generalized Vibrational Density of States (GVDOS) in Fig. 3, and the mappings shown in Fig. 5, are given in Appendix A 1. The temperature dependence of the low-lying optical bands was further investigated on the cold-neutron triple-axis spectrometer IN12 at the ILL (see Appendix A 2). Preliminary experiments were also conducted on the triple-axis spectrometer $1 \mathrm{~T}$ at the Laboratoire Léon Brillouin (LLB, France). In all INS experiments, the single crystal was mounted in a cryofurnace and aligned in the ([110]; [001]) scattering plane such that wave vectors of the form $\mathbf{Q}=\frac{2 \pi}{a}(\zeta, \zeta, \xi)$, with $a=10.7987(1)$ [13], were accessible.

Of particular importance in this work is the polarization term, which appears in the coherent one-phonon scattering 
function, $S(\mathbf{Q}, \omega)$, which in turn is proportional to the double differential inelastic neutron cross section (see Appendix A). In the case of a coherent, one-phonon scattering process by a phonon of branch $i$, with energy $\omega_{q, i}$ and polarization vector $\xi_{\omega_{q, i}}^{i}$, the neutron scattering function is written as [44]

$$
S_{p h}(\mathbf{Q}, \omega)=n(\omega) \frac{\left|F_{D}^{i}(\mathbf{Q})\right|^{2}}{\omega_{q, i}} \delta\left(\omega-\omega_{q, i}\right) \delta(\mathbf{Q}-\mathbf{q}-\mathbf{G}),
$$

where $n(\omega)=\frac{1}{1-\exp \left(-\hbar \omega / k_{B} T\right)}$ and comes from the detailed balance factor, and $\mathbf{Q}=\mathbf{q}+\mathbf{G}$ is the scattering vector given by the nearest reciprocal lattice vector $\mathbf{G}$ and the phonon wave vector q. Usually, phonons are measured in a Brillouin zone far from the $\Gamma$-point (around Bragg peaks with high Miller indices) such that $|\mathbf{G}| \gg|\mathbf{q}|$ and $\mathbf{Q} \sim \mathbf{G}$. In this work, we mainly discuss measurements performed around the Bragg peaks (006) and (222) whose moduli are much higher than $\pi / a$. The function $F_{D}^{i}(\mathbf{Q})$ in Eq. (2) is called the dynamical structure factor (DSF) and is defined as [44]

$$
F_{D}^{i}(\mathbf{Q})=\sum_{j} e^{-W_{j}(\mathbf{Q})} \frac{b_{j}}{\sqrt{M_{j}}} e^{i \mathbf{Q} \cdot \mathbf{r}_{j}}\left\{\mathbf{Q} \cdot \xi_{i}^{j}(\mathbf{Q})\right\},
$$

where $b_{j}, \mathbf{r}_{j}, M_{j}$, and $W_{j}(\mathbf{Q})$ are the coherent scattering length, fractional coordinates, mass, and Debye-Waller factor of the $j$ th element, respectively. This expression closely relates to the nuclear structure factor which determines the Bragg peak intensity. It includes an additional term, the scalar product $\left\{\mathbf{Q} \cdot \xi_{i}^{j}(\mathbf{Q})\right\} \sim\left\{\mathbf{G} \cdot \xi_{i}^{j}(\mathbf{Q})\right\}$, which contains the phonon polarization and can thus be used to distinguish longitudinal and transversal phonon modes by choosing the appropriate combination of phonon polarization wave vector and reciprocal lattice vector. The polarization vectors of longitudinal and transversal phonons are parallel and perpendicular to the phonon wave vector $\mathbf{q}$, respectively.

The lattice dynamics were simulated using a fully ordered model of a type-I clathrate with the $\mathrm{Ba}_{8} \mathrm{Ge}_{40} \mathrm{Au}_{6}$ composition in which the gold atoms occupy all the $6 c$ host sites. The periodic density functional theory (DFT) code VASP [45-47] was used for structure optimization as well as for the determination of the harmonic force constants. While the projector augmented wave method was applied for describing the ionic cores, the meta-GGA functional SCAN was used to account for exchange and correlation [34,48]. The SCAN functional has been recently proven to reproduce the phonon spectrum in type-I clathrates with a much higher accuracy. The unit cell was relaxed to the ground state using a $k$-point mesh $(5 \times 5 \times$ $5)$ centered at the zone center $(\Gamma)$ and a convergence criterion of residual forces of less than $10^{-4} \mathrm{eV} / \AA$ using a plane wave energy cutoff of $500 \mathrm{eV}$. The lattice parameter of the optimized structures obtained by the SCAN functional is of $10.78 \AA$, very close to the experimental value of $10.7987(1) \AA$ [13]. The Hellmann-Feynman forces were then calculated after introducing symmetrically non-equivalent displacements of $\pm 0.03 \AA$ in the relaxed unit cell. These forces are given as an input to the Phonopy code [49] for the calculation of the dynamical matrix.

\section{THE GRÜNEISEN PARAMETER}

Before discussing anharmonicity at the phononic level, we first address the Grüneisen parameter, $\gamma$, which is a material constant that gives an idea about the amount of anharmonicity that exists in a material. This macroscopic property provides context to our experimental findings in this paper.

The mode specific Grüneisen parameter, $\gamma_{i}$, for a phonon mode $E_{i}$ at molar volume $V$ is defined as $\gamma_{i}=-\frac{V}{E_{i}}\left(\frac{\partial E_{i}}{\partial V}\right)_{T}=$ $-\left(\frac{\partial \ln E_{i}}{\partial \ln V}\right)_{T}$. As a first approximation of the experimental Grüneisen parameter, however, we assume an averaged and temperature-dependent Grüneisen parameter for all modes, which, in the quasiharmonic approximation, depends on the volumetric thermal expansion coefficient $\alpha_{V}$, the Bulk modulus $B$, the molar volume $V$, and the specific heat at constant volume $C_{V}$ :

$$
\gamma=\frac{\alpha_{V} B V}{C_{V}} .
$$

The temperature dependence of $C_{V}(T)$ was deduced from the measurement of $C_{p}(T)$ as detailed in Appendix C. As discussed in the introduction, $C_{p}(T)$ in type-I clathrates is dominated by the contribution of the optical phonon branches and mostly by the lowest energy guest modes at $E_{1}$ such that the $\gamma$ extracted from Eq. (4) is mainly specific to the contribution of the low-lying guest modes. The change in lattice spacing with temperature in our single crystal of $\mathrm{Ba}_{7.81} \mathrm{Ge}_{40.67} \mathrm{Au}_{5.33}$ was experimentally measured by neutron Larmor diffraction on the most intense Bragg peak (006), and is plotted as black circles in Fig. 2(a). The result is compared to the miniature capacitance dilatometer measurement made by Falmbigl et al. [50] of a type-I clathrate with a similar chemical composition. Figure 2(a) shows the consistency between these two different experimental methods. The temperature dependence of the linear thermal expansion coefficient, $\alpha_{L}(\mathrm{~T})$, is then the numerical derivative of $\Delta a / a$, and the volumetric expansion coefficient is $\alpha_{V}=3 \alpha_{L}$ [see Fig. 2(b)].

The temperature-dependent molar volume for $\mathrm{Ba}_{7.81} \mathrm{Ge}_{40.67} \mathrm{Au}_{5.33}$ has been taken from the conversion of the coefficient of lattice expansion data in Fig. 2(a) to the experimental lattice parameter, and since we find no change in sound velocity within $300 \pm 150 \mathrm{~K}$ (see Fig. 3), a temperature-independent $B$ is assumed. For $\mathrm{Ba}_{7.81} \mathrm{Ge}_{40.67} \mathrm{Au}_{5.33}$, we find $B=65.60 \mathrm{GPa}$ from our measurement of the phononic sound velocities. (More details are given in Appendix B.)

The Grüneisen parameter can then be experimentally deduced from Eq. (4). It is found to be temperature independent in the range of interest for this study, with a value of $\gamma=$ 1.38. A similar method was used by Ikeda et al. [19] for $\mathrm{Ba}_{8} \mathrm{Ge}_{30} \mathrm{Ga}_{16}$ in which $\gamma_{300 \mathrm{~K}}=1.67$ was observed. In literature, the Grüneisen parameter of type-I clathrates, obtained by various methods and for different chemical compositions, is typically found to be in the range of 1.2-2.0 [16,33,50-58].

\section{EXPERIMENTAL RESULTS}

\section{A. Polarization dependence of optical branches}

The LA and TA phonon dispersions obtained from the experimental mappings (see Appendix F) of the phonon energy at 150,300 , and $530 \mathrm{~K}$ covering several Brillouin zones are shown in Figs. 3(a) and 3(b). Measurements have been performed around the most intense Bragg peak (006) in 

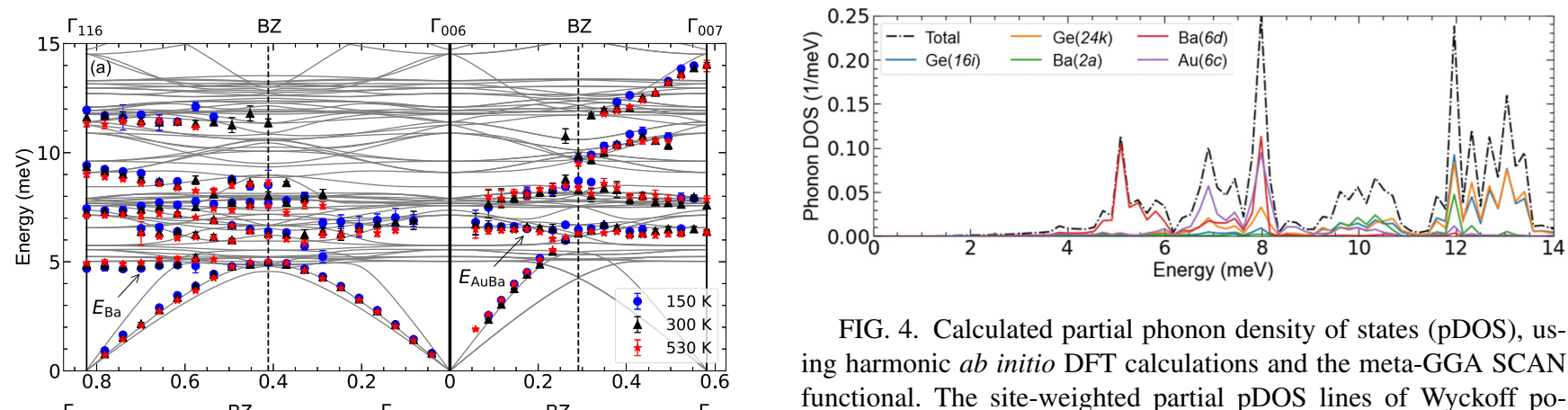

FIG. 4. Calculated partial phonon density of states (pDOS), using harmonic $a b$ initio DFT calculations and the meta-GGA SCAN functional. The site-weighted partial pDOS lines of Wyckoff positions $\mathrm{Ge}(16 i), \mathrm{Ge}(24 k), \mathrm{Ba}(2 a), \mathrm{Ba}(6 d)$, and $\mathrm{Au}(6 c)$ show their respective contributions in energy as compared to the total pDOS.

The experimental phonon spectra are compared to the simulated phonon spectrum obtained by DFT calculations using the SCAN functional (see Methods section), as shown in Figs. 3(a) and 3(b) (gray lines are the simulated phonon dispersions). A good agreement is observed on the whole spectrum especially on the transverse and longitudinal acoustic branches which are well reproduced. That corresponds to a significant improvement in the theoretical approach in comparison to the simulations performed with the PBE functional for which acoustic phonon energies are strongly underestimated $[13,15]$.

For all temperatures and both polarizations, the experimental phonon spectra exhibit an acoustic regime at low energy, which contains well-defined phonon peaks whose dispersions are delimited by low-lying optical bands. The energy

FIG. 3. (a) Transverse and longitudinal phonon dispersion curves extracted from IN5@ILL at 150 (blue circles), 300 (black triangles), and at $530 \mathrm{~K}$ (red stars) near the (006) Bragg peak. Gray solid lines depict the calculated dispersion curves. The wave vector $q$ is referenced from the zone center $(006)\left(\Gamma_{006}\right)$. Acoustic phonons with $q$ increasing towards the zone center $(116)\left(\Gamma_{116}\right)$ are mostly transversely polarized (left panel). Those with $q$ increasing towards the zone center $(007)\left(\Gamma_{007}\right)$ are mostly longitudinal (right panel). Also shown in gray is the simulated phonon spectrum. (b) Similar experimental phonon dispersions near the (222) Bragg peak, at the same three temperatures, and compared to simulations. Starting from the zone center $(222)\left(\Gamma_{222}\right)$, the transverse (left panel) polarization can be traced until zone center $(114)\left(\Gamma_{114}\right)$, while the longitudinal (right panel) polarization propagates towards the $\Gamma_{333}$. (c) Generalized vibrational density of states (GVDOS) obtained on IN5@ILL at 150 and $530 \mathrm{~K}$, along with the calculated phonon DOS (intensity has been scaled down by a factor of 3.5). All simulations correspond to harmonic $a b$ initio DFT calculations using the meta-GGA SCAN functional.

Fig. 3(a). For propagating wave vectors along the [001] direction, the longitudinal polarization is observed, while the transverse polarization for which the vibrational polarization is along the [001] direction is measured along the [110] direction. For Fig. 3(b), measurements were taken near the (222) Bragg peak, in which the longitudinal polarization is tracked along the [111] direction, and the transverse dispersion has a vibrational polarization along [111] and propagates along [112].
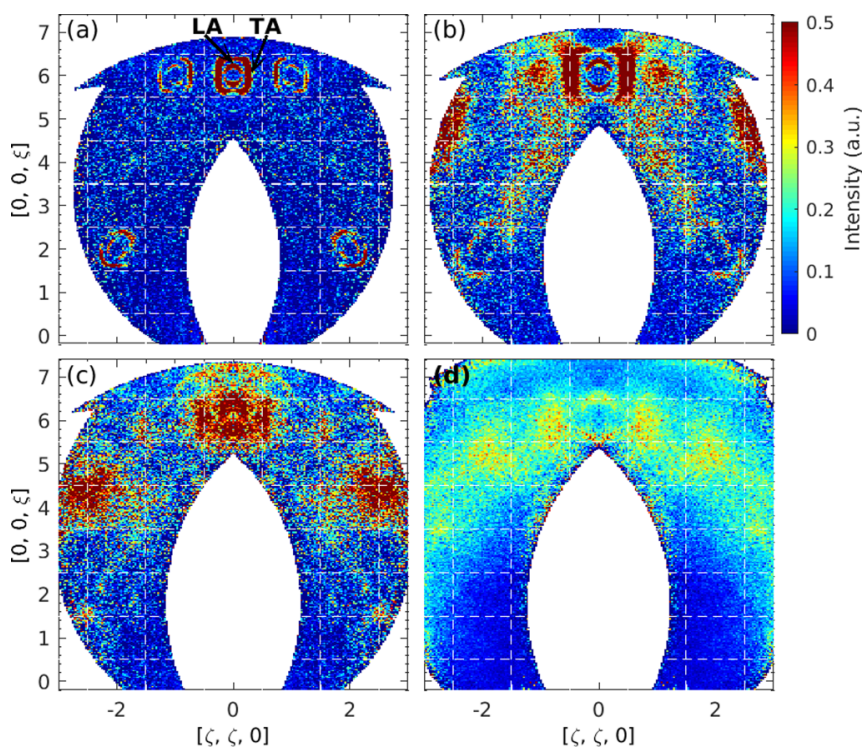

FIG. 5. Two-dimensional inelastic neutron scattering intensity distribution at fixed energy transfer in the momentum plane ([110]; [001]) for $\mathrm{Ba}_{7.81} \mathrm{Ge}_{40.67} \mathrm{Au}_{5.33}$ at $300 \mathrm{~K}$, recorded on IN5@ILL. The first three energy integrations (a)-(c) were taken at $3.5 \pm 0.15,4.8 \pm 0.15$, and $6.5 \pm 0.15 \mathrm{meV}$, respectively. (d) represents a larger integration interval of $9.5 \pm 2.5 \mathrm{meV}$. The color bar reflects a normalized intensity scale. Dashed white grids show the borders of the Brillouin zones. 
at which the acoustic dispersions are interrupted is higher for the longitudinal polarization, $6.5 \mathrm{meV}$ than for the transverse polarization, $4.8 \mathrm{meV}$. As emphasized by the use of the two sets of experimental data in Figs. 3(a) and 3(b), this is consistent across [001] and [111] longitudinal polarizations, and for [110] and [111] transverse polarizations. The cutoff effect of the acoustic branches by the low-lying optical branches is also seen in the simulated phonon spectrum. The simulation perfectly reproduces the TA dispersion, while for the longitudinal polarization, the computed acoustic branch is interrupted at $4.8 \mathrm{meV}$ and not at $6.5 \mathrm{meV}$ as observed experimentally.

Referring again to the $\mathrm{Ba}_{7.81} \mathrm{Ge}_{40.67} \mathrm{Au}_{5.33}$ crystal structure in Fig. 1(a), the motions of $\mathrm{Ba}(6 d)$ atoms in the soft plane of the large tetrakaidecahedral cages dominate the optical band centered at $4.8 \mathrm{meV}\left(E_{\mathrm{Ba}}\right)$ [59], and hybridized vibrations of $\mathrm{Au}(6 c)-\mathrm{Ba}(6 d)$ atoms are thought to dominate the optical band centered at $6.5 \mathrm{meV}\left(E_{\mathrm{AuBa}}\right)[13,29]$. This mode assignment is confirmed by the plot of the partial phonon density of states (pDOS) shown in Fig. 4. Indeed, only the $\mathrm{Ba}(6 d)$ atoms contribute in the energy range around $4.8 \mathrm{meV}$ while the optical band centered at $6.5 \mathrm{meV}$ contains contributions of the $\mathrm{Ba}(6 d)$ and the framework $\mathrm{Au}(6 c)$ atoms. For energies higher than $E_{\mathrm{Ba}, \mathrm{AuBa}}$ up to a cutoff energy of around $35 \mathrm{meV}$, the phonon spectrum consists of several broad distributions of optical bands, such as those in the range $E_{\mathrm{Ba}}-15 \mathrm{meV}$ in Fig. 3(a).

Figures 5(a)-5(c) report the two-dimensional mappings in the momentum plane ([110]; [001]) of the neutron intensity recorded at room temperature and at fixed energy of 3.5, 4.8, and $6.5 \mathrm{meV}$, respectively. These experimental mappings can be compared to DFT computed mappings of $S(\mathbf{Q}, \omega)$ in an equivalent range of momentum, shown in Figs. 6(a)-6(c). The section in momentum at $3.5 \mathrm{meV}$, in Fig. 5(a), is a cut through the acoustic branches. The Brillouin zones in which the zone center corresponds to an intense Bragg peak result in a strong dynamical structure factor of the acoustic modes and therefore contain two well-defined rings of high intensity. The intensity along the rings is not homogeneously distributed in momentum space as it is weighted by the polarization factor [described by Eq. (3)] such that when the phonon wave vector (q) is aligned/perpendicular to the Bragg wave vector $(\mathbf{G})$, the longitudinal/transverse polarization is selected. Thus, looking at the two rings surrounding the zone center $\mathbf{G}_{006}$, the outer ring which is intense along the [110] direction (perpendicular to $\mathbf{G}_{006}$ ) corresponds to TA phonons, and, reciprocally, the inner ring with maximum intensity along [001] corresponds to LA phonons. The intensity maxima along the rings follow the polarization factor and are rotated by $45^{\circ}$ between those surrounding the zone centers $\Gamma_{006}$ and $\Gamma_{222}$. This intensity distribution is reproduced on the simulated map, shown in Fig. 6(a), and carries the signature of the coherent character of the acoustic modes.

The section in momentum at a fixed energy equal to $E_{\mathrm{Ba}}$, shown in Fig. 5(b), reveals the intensity distribution of the lowest optical band which cuts the TA dispersion. It also contains the contributions of the LA modes which form the inner rings closest to the zone centers. The intensity of the optical band at $E_{\mathrm{Ba}}$ shows a distinct $\mathbf{Q}$-dependence in momentum space with intensity maxima at the zone boundaries that can be associated with the Bragg intensity at a zone center,

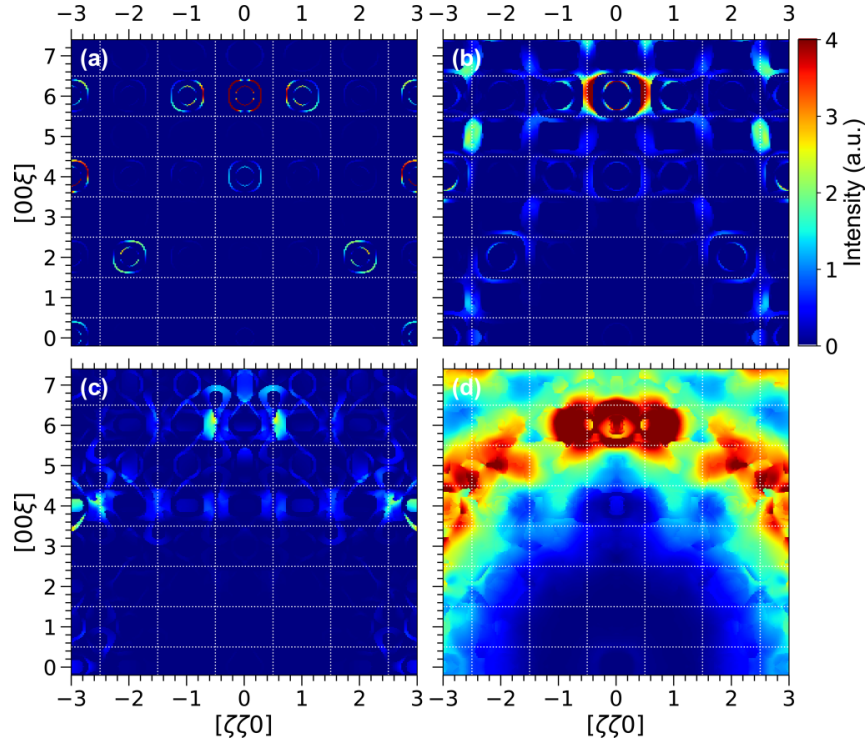

FIG. 6. Simulated two-dimensional neutron scattering function, $\mathbf{S}(\mathbf{Q}, \omega)$, at fixed energy transfer in the momentum plane ([110]; [001]) for an ideal ordered model of a type-I clathrate with the composition $\mathrm{Ba}_{8} \mathrm{Ge}_{40} \mathrm{Au}_{6}$. The first three energy integrations (a)-(c) were taken at $3.5 \pm 0.15,4.8 \pm 0.15$, and $6.5 \pm 0.15 \mathrm{meV}$, respectively. (d) represents a larger integration interval of $9.5 \pm$ $2.5 \mathrm{meV}$. The intensity in each map has been normalized to the same intensity scale, which is reflected in the color bar. Dashed white grids show the borders of the Brillouin zones.

for instance, (1.5 1.5 6) and (1.5 1.5 3.5) with Bragg peaks (006) and (222), respectively. The intensities along these maxima follow the transverse polarization and are turned by $45^{\circ}$ between the zone centers $\Gamma_{006}$ and $\Gamma_{222}$ as for the acoustic modes. This intensity distribution of the outer ring related to the optical mode at $E_{\mathrm{Ba}}$ is also reproduced in the simulated map shown in Fig. 6(b). It confirms the coherent nature of the optical modes contained in this band which thus cannot be associated with vibrations of localized and independent Einstein oscillators, in agreement with previous phonon studies on clathrates [14] and other cage compounds [60]. Indeed, an isolated Einstein oscillator-type behavior would result in a $Q^{2}$-dependent intensity distribution. Note that there are also intensity maxima at zone centers such as (116) and (114), which is not seen in the simulated map shown in Fig. 6(b) simply because the intensity is in the low range of the color scale.

Moving again in energy to $E_{\mathrm{AuBa}}$ in Fig. 5(c), one sees the maxima of intensity related to the distribution of the optical band which cuts off the LA dispersion, in addition to the ones related to $E_{\mathrm{Ba}}$ which are pinned at the zone boundary. The intensity distribution to $E_{\mathrm{AuBa}}$ around $\Gamma_{006}$ is more spread in momentum space than that at $E_{\mathrm{Ba}}$ which is also observed in the simulated map shown in Fig. 6(c). Looking carefully at the experimental map, one can distinguish minima along the [110] direction following the longitudinal polarization factor which are less obvious to see in the simulated map. The optical vibrations at $E_{\mathrm{AuBa}}$ are much less coherent than at $E_{\mathrm{Ba}}$ and exhibit the trend of being polarized longitudinally. 
TABLE I. Isobaric, isochoric, and thermal expansion (TE) rates of change in energy with increasing temperature of the lowestlying optical bands in $\mathrm{Ba}_{7.81} \mathrm{Ge}_{40.67} \mathrm{Au}_{5.33}$ (BGA) and $\mathrm{Ba}_{8} \mathrm{Ge}_{30} \mathrm{Ga}_{16}$ (BGG), extracted from the inelastic neutron scattering (INS) data shown in Figs. 7(a)-7(d), the GVDOS shown in Fig. 3, and the INS measurements of Ref. [59]. $E_{\mathrm{Ba}, \mathrm{AuBa}}$ and $E_{3,4}$ correspond to the peaks labeled in Fig. 7(e), and thermal expansion to the fit TE labeled in Fig. 7(f). Theoretical values from the Self-Consistent Phonon method, which includes the cubic and quartic contributions (SCPB), have been reported [43] and SCPB-based thermal expansion is also given, all in $1 \times 10^{-4} \mathrm{meV} / \mathrm{K}$.

\begin{tabular}{lcccccc}
\hline \hline & \multicolumn{2}{c}{ BGA } & & \multicolumn{2}{c}{ BGG } \\
\cline { 2 - 3 } \cline { 6 - 7 } \cline { 5 - 6 } & INS & GVDOS & & INS & SCPB \\
\hline$\left(\partial E_{\mathrm{Ba}} / \partial T\right)_{P}$ & 7.1 & 5.4 & & 9.3 & 11.2 \\
$\left(\partial E_{\mathrm{AuBa}} / \partial T\right)_{P}$ & - & -6.3 & & - & - \\
$\left(\partial E_{3} / \partial T\right)_{P}$ & -7.4 & -7.9 & & - & - \\
$\left(\partial E_{4} / \partial T\right)_{P}$ & -7.4 & - & & - & - \\
Thermal expansion & -2.6 & -2.7 & & -3.8 & -3.6 \\
$\left(\partial E_{\mathrm{Ba}} / \partial T\right)_{V}$ & 9.7 & 8.1 & & 13.1 & 14.8 \\
\hline \hline
\end{tabular}

Thus, the energy dispersions and momentum distributions shown in Figs. 3 and 5 demonstrate that the low-lying optical bands interact with the acoustic dispersion that is present in a given polarization. It appears that the TA dispersion couples largely with the $4.8 \mathrm{meV}$ band $\left(E_{\mathrm{Ba}}\right)$, while the LA dispersion couples mainly with the $6.5 \mathrm{meV}$ band $\left(E_{\mathrm{AuBa}}\right)$. Last, Fig. 5(d) is a experimental data integration in the interval 7-12 meV, which represents host optical band energies. A similar momentum map obtained by integrating the simulated data in the same interval is shown in Fig. 6(d). The intensity distribution of these modes on the experimental and simulated maps reveal the coherence of these host bands, which display mostly longitudinally polarized intensity.

\section{B. Temperature dependence of optical branches}

Focusing now on the temperature dependence, and in contrast to the acoustic phonon energies for which no change of their energies in temperature is observed, we clearly see sizable shifts with temperature of the optical band energies in Figs. 3(a) and 3(b). For the transverse polarization, the energy $E_{\mathrm{Ba}}$ increases upon heating, while the energies of the other optical bands, including $E_{\mathrm{AuBa}}$, follow the opposite trend. As a consequence, the temperature-dependent changes of the energy range for acoustic phonons is opposite for TA and LA phonons as it is directly related to the temperature dependence of the optical cutoff bands $E_{\mathrm{Ba}, \mathrm{AuBa}}$ [see Eq. (1)]. This is better seen by comparing the GVDOS between $150 \mathrm{~K}$ and $530 \mathrm{~K}$, depicted in Fig. 3(c). In the low-energy range below $10 \mathrm{meV}$, the GVDOS exhibits mostly three peaks at energies $E_{\mathrm{Ba}}, E_{\mathrm{AuBa}}$, and $E_{3}$. Only $E_{\mathrm{Ba}}$ follows a hardening shift upon heating, which has been similarly reported in typeI clathrates of different chemical compositions [59,61-64]. The overall structure of the measured GVDOS is reproduced well by the phonon DOS obtained from $a b$ initio simulations using the meta-GGA SCAN functional, as shown in Fig. 3(c).
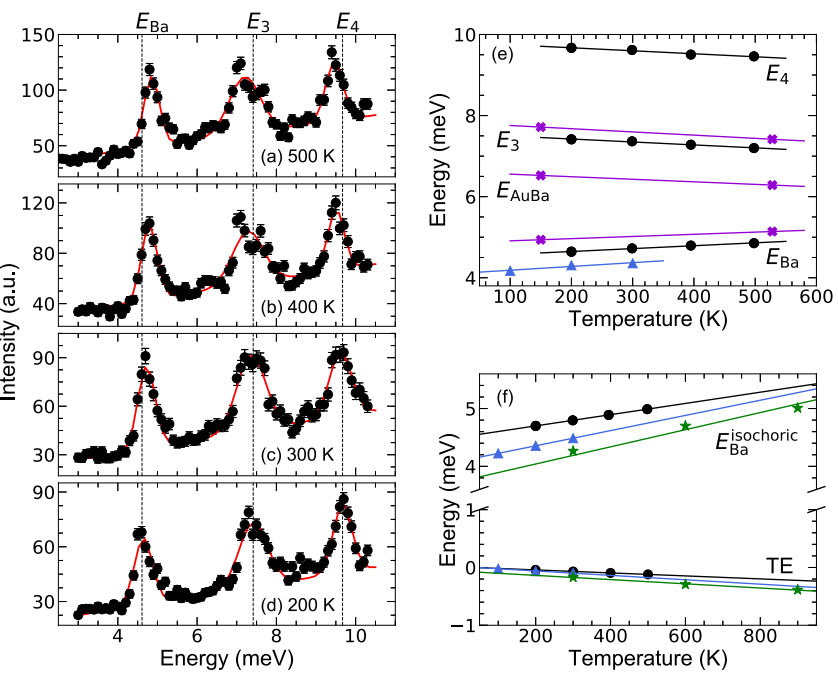

FIG. 7. (a)-(d) Inelastic neutron scattering (INS) intensity for constant $\mathbf{Q}=(113)$ scans as a function of energy (black circles) recorded on IN12@ ILL between 200-500 K for $\mathrm{Ba}_{7.81} \mathrm{Ge}_{40.67} \mathrm{Au}_{5.33}$. The solid red lines are Gaussian fits of the three optical bands whose energies are referred to as $E_{\mathrm{Ba}}$ and $E_{3,4}$. The vertical dotted black lines indicate their values at $200 \mathrm{~K}$. (e) Temperature dependence of $E_{\mathrm{Ba}}$ and $E_{3,4}$ obtained from the fits shown in panels (a)-(d) (black circles) and from the $E_{\mathrm{Ba}, \mathrm{Au}}$ and $E_{3}$ peaks in the generalized vibrational density of states shown in Fig. 3(c) (purple crosses). Blue triangles show the temperature dependence of $E_{\mathrm{Ba}}$ in $\mathrm{Ba}_{8} \mathrm{Ge}_{30} \mathrm{Ga}_{16}$ [59]. In (f), the temperature dependence of $E_{\mathrm{Ba}}$ for $\mathrm{Ba}_{7.81} \mathrm{Ge}_{40.67} \mathrm{Au}_{5.33}$ (black circles) and $\mathrm{Ba}_{8} \mathrm{Ge}_{30} \mathrm{Ga}_{16}$ (blue triangles), corrected for the thermal expansion (TE), are shown and compared to that of the $\mathrm{Ba}_{8} \mathrm{Ge}_{30} \mathrm{Ga}_{16}$ self-consistent phonon theory calculation that includes cubic and quartic terms (SCPB) [43] (green stars). The rates of TE of $\mathrm{Ba}_{7.81} \mathrm{Ge}_{40.67} \mathrm{Au}_{5.33}$ and $\mathrm{Ba}_{8} \mathrm{Ge}_{30} \mathrm{Ga}_{16}$ by INS and SCPB (same symbols/colors) are also plotted.

Some differences appear in the energy range between 5 and $7 \mathrm{meV}$, which might be linked to those observed for the LA dispersions in Figs. 3(a) and 3(b).

We then more systematically investigated these temperature dependencies by use of a triple-axis spectrometer between 200 and $500 \mathrm{~K}$. Performing energy scans at constant wave-vector $\mathbf{Q}=$ (113), where the acoustic phonon intensity is expected to be very low, allows for a more selective study of the optical branches, as seen in Figs. 7(a)-7(d). $E_{\mathrm{AuBa}}$ is not visible in this polarization. However, following the GVDOS peaks in Fig. 3(c), we find a similar $E_{3}=7.5 \mathrm{meV}$ peak, and a higher energy peak at $E_{4}=9.8 \mathrm{meV}$. The energy fits of $E_{\mathrm{Ba}}$ and $E_{3,4}$, along with $E_{\mathrm{Ba}, \mathrm{AuBa}}$ and $E_{3}$ from the GVDOS, are then plotted in Fig. 7(e). All bands display linear trends in the overall temperature range, with only $E_{\mathrm{Ba}}$ hardening with a rate of $7.0 \times 10^{-4} \mathrm{meV} / \mathrm{K}$. For comparison, the $9.3 \times 10^{-4}$ $\mathrm{meV} / \mathrm{K}$ temperature dependence of $E_{\mathrm{Ba}}$ for $\mathrm{Ba}_{8} \mathrm{Ge}_{30} \mathrm{Ga}_{16}$, measured by inelastic neutron scattering [59], has been included as well in Fig. 7(e). The rates of softening of the other optical bands in $\mathrm{Ba}_{7.81} \mathrm{Ge}_{40.67} \mathrm{Au}_{5.33}$ are summarized in Table I. 


\section{DISCUSSION}

From Eq. (1), it is evident that the reproduction of the experimentally observed $\kappa_{L}(\mathrm{~T})$ can be achieved only if the temperature dependence of the low-lying phonon modes is correctly accounted for. Therefore, we now seek direct comparison of the experimentally observed temperature dependence of the $E_{\mathrm{Ba}}$ band to results from SCP theory. The combined cubic and quartic anharmonicity terms give the isochoric contribution of the thermal change of phonon energy, $\left(\frac{\partial E}{\partial T}\right)_{V}$, while experimental measurements, such as the ones presented above, are usually performed at constant pressure and give access to the isobaric thermal variation, $\left(\frac{\partial E}{\partial T}\right)_{P}$. To the first order, the thermodynamic relation between those quantities is given by

$$
\left(\frac{\partial E}{\partial T}\right)_{V}=\left(\frac{\partial E}{\partial T}\right)_{P}+E(T) \alpha_{V}(T) \gamma,
$$

where the far right term corresponds to the mode-specific thermal expansion which we have experimentally quantified in $\mathrm{Ba}_{7.81} \mathrm{Ge}_{40.67} \mathrm{Au}_{5.33}$ and $\mathrm{Ba}_{8} \mathrm{Ge}_{30} \mathrm{Ga}_{16}$ through their volumetric thermal expansion coefficients, $\alpha_{V}(\mathrm{~T})$, average Grüneisen parameters, $\gamma$, and energies of the mode under investigation (with the help of $\alpha_{V}$ and inelastic neutron scattering measurements in Refs. [19,59] for $\mathrm{Ba}_{8} \mathrm{Ge}_{30} \mathrm{Ga}_{16}$ ). While experimentally we cannot further separate the cubic from the quartic anharmonic term, we discuss a conceptual first approximation in Appendix E. In Table I the isochoric dependencies of $\mathrm{Ba}_{7.81} \mathrm{Ge}_{40.67} \mathrm{Au}_{5.33}$ and $\mathrm{Ba}_{8} \mathrm{Ge}_{30} \mathrm{Ga}_{16}$, shown in Fig. 7(f), are deduced using Eq. (5) and thermal expansion contributions. For the lowest optical band at $E_{\mathrm{Ba}}$, anharmonicity accounts for $9.7 \times 10^{-4} \mathrm{meV} / \mathrm{K}$ for $\mathrm{Ba}_{7.81} \mathrm{Ge}_{40.67} \mathrm{Au}_{5.33}$, and $13.1 \times 10^{-4} \mathrm{meV} / \mathrm{K}$ for $\mathrm{Ba}_{6} \mathrm{Ge}_{30} \mathrm{Ga}_{16}$. The difference between $\mathrm{Ba}_{7.81} \mathrm{Ge}_{40.67} \mathrm{Au}_{5.33}$ and $\mathrm{Ba}_{8} \mathrm{Ge}_{30} \mathrm{Ga}_{16}$ can be attributed to difference of the $\mathrm{Ba}$ local environment in the tetrakaidecahedral cages.

Conversely, for the SCP simulations, the SCP calculation which includes the quartic and cubic contributions (SCPB) with a rate of $14.8 \times 10^{-4} \mathrm{meV} / \mathrm{K}$ can be used along with $\gamma^{\mathrm{SCPB}}(T)$ in order to find the equivalent isobaric rate of $11.2 \times 10^{-4} \mathrm{meV} / \mathrm{K}$. (More details are given for SCPB in Appendix D.) We therefore find close isochoric matching between SCPB and experimentally deduced anharmonicity in $\mathrm{Ba}_{8} \mathrm{Ge}_{30} \mathrm{Ga}_{16}$, allowing us to experimentally validate the SCPB method in clathrates.

\section{CONCLUSIONS AND PERSPECTIVES}

In summary, we confirm the good agreement between our measurements and the $a b$ initio harmonic calculations using the meta-GGA SCAN functional of the overall phonon spectrum along different directions and for the transverse and longitudinal polarizations on the type-I clathrate $\mathrm{Ba}_{7.81} \mathrm{Ge}_{40.67} \mathrm{Au}_{5.33}$. However, experimentally, the TA and LA branches are delimited by two optical phonon bands of different nature which is not reproduced by our simulations. While the former hybridizes with transverse optical vibrations centered at $E_{\mathrm{Ba}}=4.8 \mathrm{meV}$ associated with the coherent guest motions of $\mathrm{Ba}(6 d)$ in the soft plane of the tetrakaidecahedral cages, the latter is interrupted by the longitudinal optical band centered at $E_{\mathrm{AuBa}}=6.5 \mathrm{meV}$ related to the coherent hybridized motions of the substituted $\mathrm{Au}(6 c)$ host and the $\mathrm{Ba}(6 d)$ guest atoms. The TA dispersion is perfectly reproduced by our $a b$ initio simulation in the whole Brillouin zone. On the other hand, a difference appears on the longitudinal branch in the region of the phase space where optical and acoustic modes hybridize such that the LA branch is predicted to be cut off at the same energy as the transverse one.

Upon cooling, $E_{\mathrm{AuBa}}$ increases, following a rate of change guided by thermal expansion, while $E_{\mathrm{Ba}}$ decreases. The experimental isochoric rate of change of $E_{\mathrm{Ba}}$ extracted in $\mathrm{Ba}_{8} \mathrm{Ge}_{30} \mathrm{Ga}_{16}$, which quantifies the amount of cubic and quartic anharmonicity, is in good agreement with the SCPB simulations in $\mathrm{Ba}_{8} \mathrm{Ge}_{30} \mathrm{Ga}_{16}$. In $\mathrm{Ba}_{7.81} \mathrm{Ge}_{40.67} \mathrm{Au}_{5.33}$, a much lower rate of change is found which is assumed to originate from the difference of the defect structure. On the other hand, the difference between the transverse and the longitudinal cutoff energies observed experimentally, which is not seen in the simulations, indicates a more subtle polarization dependent mechanism involving either the disorder and/or the anharmonic polarization mixing whose consideration in the SCP-like approach will surely reveal an improved $\kappa_{L}(T)$ dependence, especially in the intermediate temperature range as seen in Fig. 1(b).

Data from inelastic neutron scattering measurements at the ILL are available at [65], and LLB measurements correspond to proposal 657 .

\section{ACKNOWLEDGMENTS}

S.R.T. acknowledges financial support from the ISP program of the IDEX Université Grenoble Alpes. S.P. acknowledges support from the Lyon IDEX Scientific Breakthrough program for funding of the project IPPON. This work has been carried out within the European C-MetAC network [66]. The authors would like to thank T. Tadano, N. Vast, and T. Nakayama for fruitful discussions.

\section{APPENDIX A: INELASTIC NEUTRON SCATTERING MEASUREMENTS}

A neutron scattering event involves incident neutrons with initial energy and wave vector $\left(\left|E_{i}, \mathbf{k}_{i}\right\rangle\right)$ impinging on a sample and being scattered, resulting in scattered neutrons with $\left(\left|E_{f}, \mathbf{k}_{f}\right\rangle\right)$. An inelastic neutron scattering (INS) experiment measures the amount of flux of the initial neutron beam that has been scattered into the solid angle element of interest, $d \Omega_{f}$, within energy range of interest $d E_{f}$. The cross section, $\sigma$, is defined as the number of neutrons scattered per second out of the number of incident neutrons per $\mathrm{cm}^{2}$ per second. The double differential of $\sigma$ for neutron scattering, $\frac{d^{2} \sigma}{d \Omega_{f} d E_{f}}$, can be related to the coherent inelastic scattering function, $S(\mathbf{Q}, \omega)$, by $\frac{d^{2} \sigma}{d \Omega_{f} d E_{f}}=N \frac{k_{f}}{k_{i}} S(\mathbf{Q}, \omega)$, where $\mathrm{N}$ is the number of nuclei $[44,67]$.

\section{Time-of-flight spectroscopy}

Inelastic neutron scattering measurements depicted in Figs. 3 and 5 of the main text and Figs. 12-18 in Appendix F were made using cold-neutron time-of-flight spectroscopy. The time-of-flight measurements took place on IN5@ILL 
TABLE II. For the given experimental conditions on IN5@ILL, the components of $q$ resolution are summarized. Values were calculated for dispersions near the (006) Bragg peak in the momentum plane ([110]; [001]) and are categorized by horizontal resolutions for the primary, [110], and secondary, [001], axes of the scattering plane, followed by the vertical resolution for the out-of-plane direction, [110]. Pixel/step divergence refers to the divergence due to the size of the IN5 detector tubes as seen from the sample distance and to the step size of $0.5^{\circ}$ chosen for this particular experiment for the [110] direction. Beam divergence refers to the divergence that occurs due to the IN5 neutron guide. Values are given in $\AA^{-1}$.

\begin{tabular}{lcc}
\hline \hline & Pixel/step divergence & Beam divergence \\
\hline Along [001] & $5.66 \times 10^{-3}$ & $9.96 \times 10^{-3}$ \\
Along [110] & $3.06 \times 10^{-2}$ & $3.92 \times 10^{-2}$ \\
Along [110] & $1.25 \times 10^{-2}$ & $3.29 \times 10^{-2}$ \\
\hline \hline
\end{tabular}

using an incident neutron wavelength of $\lambda=3.2 \AA$ and a cryofurnace. Scans covered an $\Omega$ range of $54^{\circ}$, with a sample rotation of $0.5^{\circ}$ in between each scan. Time-of-flight data were reduced using Mantid [68] and then processed into a four-dimensional $S(\mathbf{Q}, E)$ file and further analyzed with the Horace package [69] under Matlab.

Considerable effort was made to understand $q$ and $E$ resolution $(d q, d E)$ of the instrument in order to make realistic data integrations with Horace. The overall instrumental resolution is governed by the incoming neutron beam energy and monochromatization, the beam divergence in horizontal and vertical directions, the sample mosaic, the receiving slit sizes in front of the detector, and the final neutron energy [70]. This then must be compared to the minimum step size that can be achieved with the instrument, as explained hereafter.

By taking into account the divergence due to the size of the detector tubes of IN5@ILL and the beam divergence while in the $\lambda=3.2 \AA$ condition, we have defined the resolution for the [001] and [110] directions while near the (006) Bragg peak. More specifically, detector tubes on IN5 have a diameter of $2.54 \mathrm{~cm}$, meaning that with a distance of $4 \mathrm{~m}$ between the sample and detectors, there is a beginning divergence of $0.36^{\circ}$ due to instrumental conditions. To this we add the corresponding horizontal and vertical beam divergences, $0.64^{\circ}$ and $0.96^{\circ}$, respectively, due to the incident neutron wavelength and neutron guide horizontal and vertical super-mirror indices on IN5 [70]. Using these starting points, we then calculated the local resolution limits around our Bragg peak of interest, the (006). These are summarized in Table II.

Next, as a first approximation, we consider that $q$ and $E$ are decoupled for a time-of-flight spectrometer, unlike on a triple-axis spectrometer. This means that the effective phonon energy resolution depends on the effect of energy broadening due to the reciprocal space, $\Delta E_{\text {sound vel, }}$ and on the instrumental resolution for a given energy transfer, $\Delta E_{\text {instr }}$. The latter has been calculated using the incident neutron wavelength, speed of the IN5@ILL choppers (12 000 rpm), and additional spectrometer parameters [70]. For our given experimental conditions, Fig. 8 depicts the energy resolution as it changes with the energy of the scattering event. The former, on the other hand, refers to $\Delta E_{\text {sound vel }}=v_{s} \Delta q$, where $v_{s}$ is the sound velocity of the particular dispersion

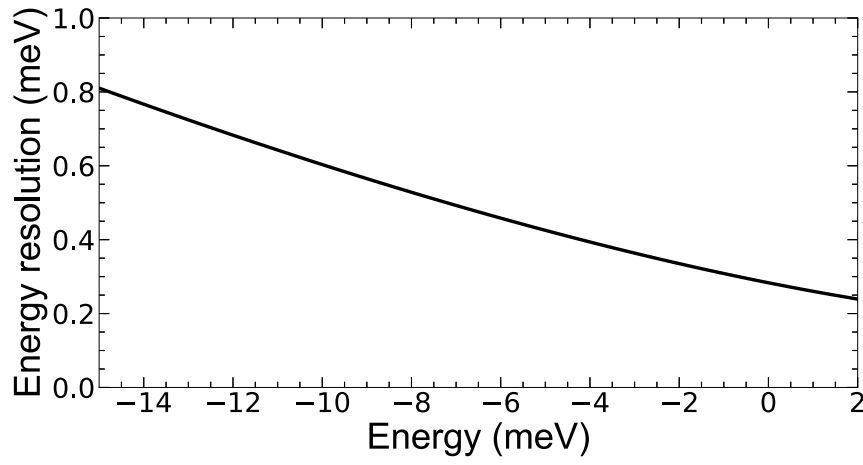

FIG. 8. The energy resolution, given our specific experimental conditions, of the cold-neutron time-of-flight spectrometer IN5@ILL is plotted in the energy range of interest for results discussed in this article. The calculation is made by considering the incident neutron wavelength, chopper speed, and other instrumental parameters specific to IN5. The equation for calculating energy resolution is derived in Ref. [70].

of interest. For $\mathrm{LA}_{001}$ near the (006) Bragg peak with $v_{s}=$ $25.84 \mathrm{meV} \AA$, the resulting $\Delta E_{\text {sound vel }}=0.30 \mathrm{meV}$, while for $\mathrm{TA}_{110}^{001}$ with $v_{s}=16.99 \mathrm{meV} \AA, \Delta E_{\text {sound vel }}=0.25 \mathrm{meV}$. The total effective phonon energy resolution is therefore $\sqrt{\left(\Delta E_{\text {sound vel }}\right)^{2}+\left(\Delta E_{\text {instr }}\right)^{2}}$.

The minimum step size that can be chosen when preparing the 4D data matrix in Horace is therefore given by the step sizes due to detector pixel sizes, step rotation, and step size in energy. These steps are generally smaller than the effective instrumental resolution. It should be noted that $d q_{T}$, or the transversal $d q$ step size, is strongly $Q$ dependent, since it is equal to $\Delta$ (rotation) $\times Q$, where $\Delta$ (rotation) is the rotation step size expressed in radians. As stated above, there is a $0.5^{\circ}$ step size for these experimental data. The final Horace integration limits are derived from Table II, in which the overall $d q$ resolution is: $\sqrt{\left(\Delta q_{\text {step div. }}\right)^{2}+\left(\Delta q_{\text {beam div. }}\right)^{2}}$.

Figure 12 contains two-dimensional plots showing the energy spectrum in a given direction within the momentum plane were cut and then folded along symmetries using the appropriate Horace functions. For subplots (a), (c), and (e), $d q=0.018$ r.l.u. for $0-5.75 \mathrm{meV}, 0.036$ r.l.u. for 5.75 $6.3 \mathrm{meV}$, and 0.072 for $6.3-15 \mathrm{meV}$, while $d E=0.075 \mathrm{meV}$ between 0 and $5.75 \mathrm{meV}$ and 0.1 for $5.75-15 \mathrm{meV}$. For subplots (b), (d), and (f), $d q=0.037$ r.l.u., and $d E=0.075 \mathrm{meV}$ between 0 and $7 \mathrm{meV}$, and $0.1 \mathrm{meV}$ between 7 and $15 \mathrm{meV}$. The energy is then squared in order to display $S(q, E) \times E^{2}$ in the final subplots of Fig. 12.

The Fig. 5 subplots reflect intensity seen across the measured part of the momentum plane ([110];[001]) at a given energy. Each pixel on the surface plot is a $0.03 \times 0.03$ r.l.u. square. Subplots (a)-(c) were made using an energy integration of $\mathrm{E} \pm 0.15 \mathrm{meV}$, where $E$ is $4.8,6.5,7.5$ in the cases of subplots (a)-(c), and for subplot (d), $9.5 \pm 2.5 \mathrm{meV}$. Similar cut and folded symmetry Horace functions were used.

In Fig. 3(a) 1D scans around the intense (006) Bragg peak along transverse and longitudinal polarizations (see Appendix F) were once again cut using Horace functions. A tight data integration along the propagation axis was chosen in order to selectively measure along a given polarization in a focusing condition. For Figs. 13(a), 14(a), and 15(a), 
$d q=0.018$ r.l.u. along the propagation axis [ $\zeta \zeta 0], 0.04$ r.l.u.

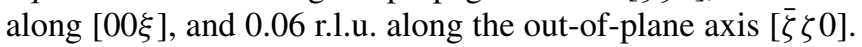
For Figs. 13(b), 14(b), and 15(b), $d q=0.037$ r.l.u. along the

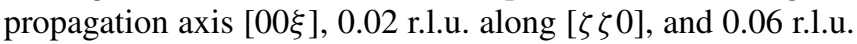
along $[\bar{\zeta} \zeta 0]$.

For the one-dimensional scans around the (222) Bragg peak in Fig. 3(b), the same procedure as described above has been completed to reflect the resolution limits near this new position in reciprocal space. As there is less intensity in this region, however, integrations slightly larger than those of the strict minimums were taken: $d q=0.08$ r.l.u. along the propagation axis $[\zeta \zeta 0], 0.08$ r.l.u. along [00 $\xi$ ], and 0.06 r.l.u. along the out-of-plane axis $[\bar{\zeta} \zeta 0]$. The $1 \mathrm{D}$ scans used to create Fig. 3(b) are shown in Figs. 16(a) and 16(b), Figs. 17(a) and 17(b), and Fig. 18(a) and 18(b).

In addition, high-resolution time-of-flight measurements were taken with $\lambda=4.8 \AA$ in order to obtain the neutronweighted generalized vibrational density of states (GVDOS) plot in Fig. 3(c), calculated by the MUPHOCOR (MUltiPHOnon CORrection) routine [71] in the LAMP program [72] for single crystal data. Scans covering an $\Omega$ range of $46^{\circ}$ at $150 \mathrm{~K}$ and $34^{\circ}$ at $530 \mathrm{~K}$, both with sample-rotation step sizes of $2^{\circ}$, were used to make the calculation, and the atomic mass and expected neutron scattering cross section of $\mathrm{Ba}_{7.81} \mathrm{Ge}_{40.67} \mathrm{Au}_{5.33}$ were used as initial starting parameters.

\section{Triple-axis spectroscopy}

Figures 7(a)-7(d) reflect the measurements taken on the cold-neutron triple-axis spectrometer (TAS) IN12@ILL. The experiment was conducted with a fixed $k_{f}=1.55 \AA^{-1}$, and with the $k_{i}$ velocity selector, $k_{f}$ Be filter, and cryofurnace options. The three optical bands shown in subplots (a)-(d) were fitted as Gaussian peaks because the signal is not a single phonon but a distribution of optical branches.

Preliminary experiments, performed on the triple-axis spectrometer 1T@LLB with a fixed $k_{f}=2.662 \AA^{-1}$, were extremely important in this work. Indeed, the polarization dependence of the low-lying optical phonon band at $E_{\mathrm{Ba}}$ was formerly observed in these experiments.

\section{Neutron Larmor diffraction}

Neutron Larmor diffraction takes advantage of the neutron resonance spin echo technique for triple-axis spectrometers in order to measure the change in lattice spacing with temperature with extreme sensitivity $\left(1.5 \times 10^{-6}\right)$ [73-76]. Such a measurement was made on the (006) Bragg peak position between 3 and $300 \mathrm{~K}$, and is shown in Fig. 9(a) (also see Fig. 2). Measurements were taken in increments of $5 \mathrm{~K}$ with a fixed $k_{i}$ of $2.13 \AA^{-1}$ on the thermal-neutron triple-axis spectrometer TRISP@FRM-II.

\section{APPENDIX B: CALCULATION OF BULK MODULUS FROM ELASTIC CONSTANTS}

Given that we have measured acoustic phonons in several high-symmetry directions, we can use the sound velocities to estimate certain mechanical properties such as the bulk modulus, $B$. Sound velocities of transverse, $v_{\mathrm{TA}}$, and longitudinal, $v_{\mathrm{LA}}$, polarizations can be related to the single crystal elastic
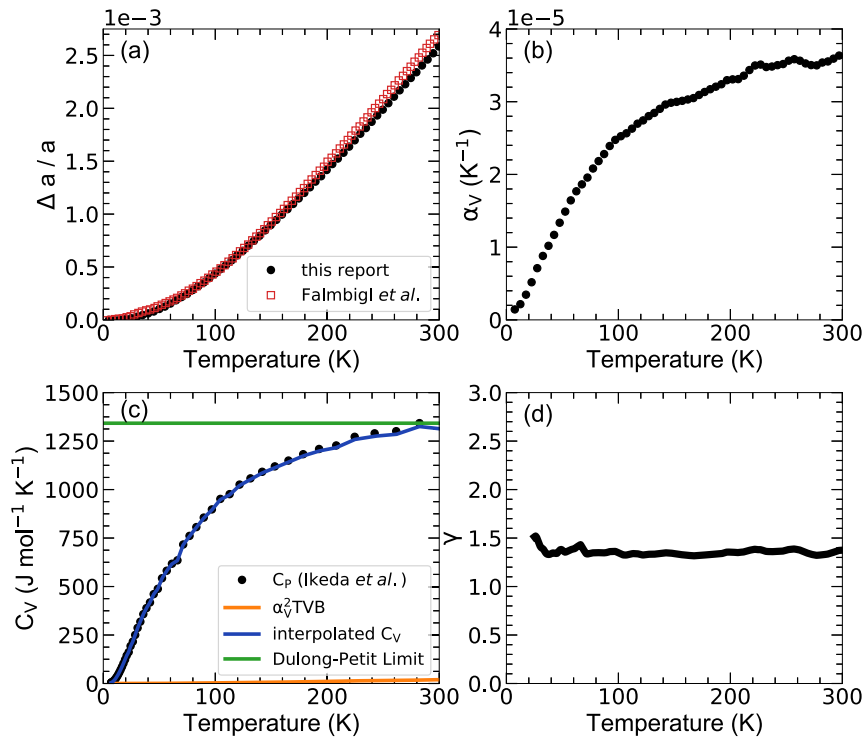

FIG. 9. The temperature-dependent Grüneisen parameter $(\gamma)$ in (d) for $\mathrm{Ba}_{7.81} \mathrm{Ge}_{40.67} \mathrm{Au}_{5.33}$ is calculated using the rate of thermal expansion $(\Delta a / a)$ in (a), the volumetric coefficient of thermal expansion $\left(\alpha_{V}\right)$ in (b), the constant volume specific heat in (c), and the bulk modulus calculated in Appendix B. The rate of thermal expansion has been measured by Larmor diffraction on TRISP@FRM-II (black circles), and compared to that of Falmbigl et al. (empty red squares) [50]. Its numerical derivative is used to find $\alpha_{L}=\alpha_{V} / 3$. The measurement of $C_{P}$ (black circles) from Ikeda et al. [19] was used to calculate $C_{V}$ (solid blue line).

constants at $0 \mathrm{~K}, c_{i j}$, through the following [16,77-79]:

$$
\begin{aligned}
& v_{\mathrm{LA}_{001}}=\sqrt{\frac{c_{11}}{\rho}}, v_{\mathrm{TA}_{110}^{001}}=\sqrt{\frac{c_{44}}{\rho}}, \\
& v_{\mathrm{TA}_{112}^{111}}=\sqrt{\frac{c_{11}-c_{12}+c_{44}}{3 \rho}} .
\end{aligned}
$$

For $\mathrm{Ba}_{7.81} \mathrm{Ge}_{40.67} \mathrm{Au}_{5.33}$, we find $v_{\mathrm{LA}_{001}}=3,926 \mathrm{~m} / \mathrm{s}$, $v_{\mathrm{TA}_{110}^{001}}=2,582 \mathrm{~m} / \mathrm{s}$, and $v_{\mathrm{TA}} \frac{111}{112}=2,234 \mathrm{~m} / \mathrm{s}$ at $300 \mathrm{~K}$. Therefore, $c_{11}=103.15 \mathrm{GPa}, c_{44}=44.61 \mathrm{GPa}$, and $c_{12}=47.49 \mathrm{GPa}$ at $0 \mathrm{~K}$. Okamoto et al. have experimentally measured $c_{i j}$ for $\mathrm{Ba}_{8} \mathrm{Ge}_{30} \mathrm{Ga}_{16}$ and found $c_{i j}^{300 \mathrm{~K}}$ within $10 \%$ of our values [80]. Similar values have been found for other type-I clathrates as well [81].

Using these elastic constants, we can calculate the material bulk modulus at $0 \mathrm{~K}, B$, through Eq. (B2), which gives us $B=$ $65.60 \mathrm{GPa}$. Again, the same value is found for $\mathrm{Ba}_{8} \mathrm{Ge}_{30} \mathrm{Ga}_{16}$ with almost no temperature dependence $[19,80]$ :

$$
B=\frac{1}{\chi}=\sqrt{\frac{c_{11}+2 c_{12}}{3} .}
$$

\section{APPENDIX C: CALCULATION OF EXPERIMENTAL GRÜNEISEN PARAMETER (CONTINUED)}

This section expands on the experimental Grüneisen parameter results discussed in the main text, in particular on Eq. (4). Figures 9(a) and 9(b) have already been presented in Fig. 2. From Ikeda et al. [19] we have experimental $C_{P}$ for $\mathrm{Ba}_{7.81} \mathrm{Ge}_{40.67} \mathrm{Au}_{5.33}$. To calculate $C_{V}$, we use the relation 
$C_{P}=C_{V}+\alpha_{V}^{2} T V B$ [82], and this is plotted in Fig. 9(c). Finally, using the given equation for calculating the average Grüneisen parameter, $\gamma(T)$ is calculated and plotted in Fig. 9(d). This method of experimental deduction gives $\gamma_{300 \mathrm{~K}}$ $=1.38$, which is temperature independent within the temperature range of study.

\section{APPENDIX D: COMPARING TO SCP METHOD CALCULATIONS}

There are two different calculations of the $E_{\mathrm{Ba}}$ optical band in $\mathrm{Ba}_{8} \mathrm{Ge}_{30} \mathrm{Ga}_{16}$ that have been summarized by Tadano and Tsuneyuki in the Supplementary Material of Ref. [43]: (1) SCP: SCP method calculations in which the real part of the quartic anharmonic term, or the Loop free energy diagram, determines phonon frequency renormalizations in the system, and (2) SCP+Bubble: the same calculation, but made by also including the real part of the cubic anharmonic term, the Bubble free energy diagram. (The second calculation will be referred to as SCPB in this discussion.) We therefore reflect that the SCPB calculation directly provides us with $\left(\frac{\partial E}{\partial T}\right)_{V}$ of $E_{\mathrm{Ba}}$ and that the self-energy terms in the SCPB calculation self-consistently include the renormalized phonon energies including the effect of the quartic anharmonicity. (This is an important distinction for Appendix E, as this means that we cannot extract the harmonic Bubble term but only the self-consistent Bubble term.)

In order to move between isobaric and isochoric representations of the SCPB calculation discussed in the main text, we have calculated the SCPB-based thermal expansion: $E^{\mathrm{SCPB}}(T) \alpha_{V} \gamma^{\mathrm{SCPB}}(T)$. In this way, the determination of the thermal expansion is much more exact than by using the quasiharmonic approximation for which only the harmonic phonon energies are considered. As will be shown in the following paragraphs, $\gamma^{\mathrm{SCPB}}(T)$ and $E^{\mathrm{SCPB}}(T)$ come from the information given in the Supplementary Material of Ref. [43], and as a first approximation we use the experimental $\alpha_{V}$ for $\mathrm{Ba}_{8} \mathrm{Ge}_{30} \mathrm{Ga}_{16}$ from Ikeda et al. [19].

The isochoric rate of change in energy of $E_{\mathrm{Ba}}$ with temperature for the SCP calculation is $17.9 \times 10^{-4} \mathrm{meV} / \mathrm{K}$, and $14.8 \times 10^{-4} \mathrm{meV} / \mathrm{K}$ for the SCPB calculation. Next, with the use of the mode-specific definition of the Grüneisen parameter, $\gamma_{i}=-\frac{V}{E_{i}}\left(\frac{\partial E_{i}}{\partial V}\right)=-\left(\frac{\partial \ln E_{i}}{\partial \ln V}\right)_{T}$, the SCPB-based Grüneisen parameter for the $E_{\mathrm{Ba}}$ mode can be calculated using Fig. 10(b). The mode-specific Grüneisen parameter for the SCP calculation [Fig. 10(a)] is 4.71, 2.57, 2.06, and 1.76 for temperatures of $0,300,600$, and $900 \mathrm{~K}$, respectively, and in similar fashion for the SCPB calculation [Fig. 10(b)]: 4.81, 2.98, 2.34, and 1.94. Although these Grüneisen parameters appear to be slightly larger than the average clathrate experimental values discussed in the main text, we note that these parameters reflect the anharmonicity found specifically in the $E_{\mathrm{Ba}}$ optical band. With these mode-specific Grüneisen parameter results, the SCPB thermal expansion shown in Fig. 7(f) was calculated and, by extension, the isobaric form of the SCPB calculation was found to have a rate of change of $11.2 \times 10^{-4} \mathrm{meV} / \mathrm{K}$.

We also note that there are experimental rates of change for $\mathrm{Ba}_{8} \mathrm{Ge}_{30} \mathrm{Ga}_{16}$ that match the isochoric SCP (no cubic component) calculation. The Raman measurements of Takasu et al. [61-63], with a rate of change of $17.1 \times 10^{-4} \mathrm{meV} / \mathrm{K}$, cause

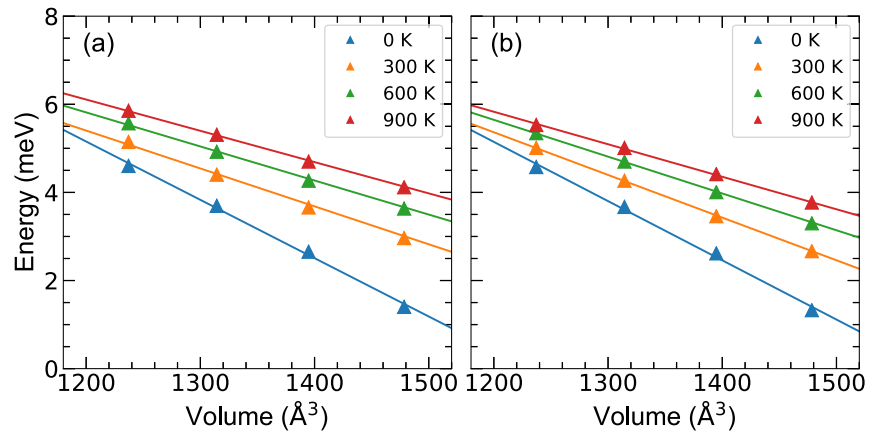

FIG. 10. Calculations from Table S2 of Ref. [43] are plotted in order to obtain $\left(\frac{\partial E_{j}}{\partial V}\right)_{T}$ for the $E_{\mathrm{Ba}}$ optical band, namely, the rate of change in volume of $\mathrm{Ba}_{8} \mathrm{Ge}_{30} \mathrm{Ga}_{16}$ with energy for "SCP" (a) and "SCP+Bubble" (b).

a discrepancy between Raman measurements of $\mathrm{Ba}_{8} \mathrm{Ge}_{30} \mathrm{Ga}_{16}$ measured by this group and by those of Christensen et al. [59]. We emphasize, however, that the isobaric $17.1 \times 10^{-4}$ $\mathrm{meV} / \mathrm{K}$ should not be compared to the isochoric quartic-only calculation.

\section{APPENDIX E: FIRST APPROXIMATION DECOUPLING OF CUBIC AND QUARTIC ANHARMONICITY}

Even though we cannot definitively isolate the cubic and quartic anharmonicity terms from an inelastic neutron scattering $\left(\frac{\partial E}{\partial T}\right)_{P}$ measurement, we will attempt to qualitatively interpret their weighted importance on $E_{\mathrm{Ba}}$.

The generalized vibrational density of states (GVDOS), as seen in Fig. 3(c), represents the isobaric temperature dependence of $\mathrm{Ba}_{7.81} \mathrm{Ge}_{40.67} \mathrm{Au}_{5.33}$. However, if the energy axis of the data at $530 \mathrm{~K}$ is scaled by $3 \%$ of the original values, as seen in Fig. 11, then globally, all peaks except for $E_{\mathrm{Ba}}$ now align. This is already a powerful conclusion about the uniqueness of $E_{\mathrm{Ba}}$, pointing to the strong anharmonicity that governs its behavior as opposed to all other higher energy peaks, for which one scaling factor can explain the complete temperature dependencies.

This $3 \%$ scaling factor can be understood to be made up of the thermal expansion and cubic components, since

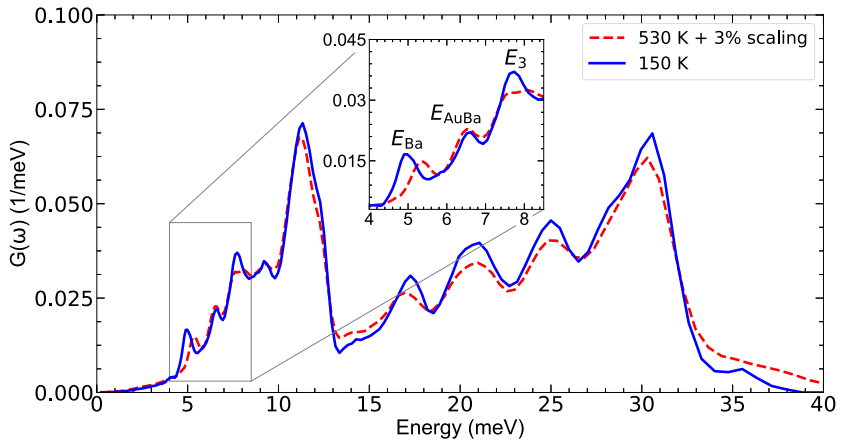

FIG. 11. Generalized vibrational density of states obtained from the data collection recorded on IN5@ILL at 150 and $530 \mathrm{~K}$. The energy axis of the data at $530 \mathrm{~K}$ has been scaled by $3 \%$, leading to an alignment of all but the lowest peaks. 
we will assume that the quartic term is localized onto only $E_{\mathrm{Ba}}$ as a first approximation. This simplification is supported by Tadano and Tsuneyuki [43], who find that the quartic anharmonic phonon energy renormalizations of the SCP (not SCPB; see Appendix D for more details) calculation are focused onto modes below $9.92 \mathrm{meV}\left(80 \mathrm{~cm}^{-1}\right)$ for $\mathrm{Ba}_{8} \mathrm{Ge}_{30} \mathrm{Ga}_{16}$. We therefore extrapolate to say that modes higher than $E_{\mathrm{Ba}}$ are controlled by only thermal expansion and the cubic term, while $E_{\mathrm{Ba}}$ has contributions from thermal expansion, the cubic term, and the quartic term.

Let us first look at GVDOS peaks between 10 and $35 \mathrm{meV}$, for which we must consider only thermal expansion and cubic components. To reiterate, by studying the $3 \%$ scaling factor, we are studying the isobaric rate of change for peaks between 10 and $35 \mathrm{meV}$. We recall that, using Eq. (5), the peak-specific thermal expansion for each of the GVDOS peaks between 10 and $35 \mathrm{meV}$ in Fig. 11 can be calculated, and the result is that thermal expansion consistently accounts for $45 \%-50 \%$ of the total isobaric rate of change. Given our assumption that these modes are controlled only by the thermal expansion and cubic terms, this leaves a remaining $50 \%-55 \%$ of the $3 \%$ scaling which must be understood as the rate of change of the cubic anharmonicity. In this manner, the 3\% scaling factor of peaks between 10 and $35 \mathrm{meV}$ is fully accounted for.

Expanding on this $3 \%$ scaling concept to $E_{\mathrm{Ba}}$, we recall the values given in Table I: $E_{\mathrm{Ba}}$ has an isobaric rate of change of $5.4 \times 10^{-4} \mathrm{meV} / \mathrm{K}$ and a thermal expansion rate of change of $-2.7 \times 10^{-4} \mathrm{meV} / \mathrm{K}$, giving the isochoric rate of change of $8.1 \times 10^{-4} \mathrm{meV} / \mathrm{K}$. However, this time we expect for the isobaric rate of change to reflect thermal expansion, cubic, and quartic contributions. As stated above, the rate of thermal expansion for $E_{\mathrm{Ba}}$ is already known, leaving the cubic and quartic contributions, for which we also know the total isochoric (cubic and quartic) contribution. However, after the 3\% scaling shown in Fig. 11, the rate of change for $E_{\mathrm{Ba}}$ becomes $9.4 \times 10^{-4} \mathrm{meV} / \mathrm{K}$. Therefore, the difference between the $3 \%$ scaling factor and isochoric rate of change, which is $-1.3 \times$ $10^{-4} \mathrm{meV} / \mathrm{K}$, must be due to cubic anharmonicity, meaning that $9.4 \times 10^{-4} \mathrm{meV} / \mathrm{K}$ is the quartic contribution to $E_{\mathrm{Ba}}$.

While we cannot directly compare the Bubble contribution of the "SCP+Bubble" (SCPB) calculation (see Appendix D) to the cubic contribution using this GVDOS rescaling method, we note that $-1.3 \times 10^{-4} \mathrm{meV} / \mathrm{K}$ has the correct sign for the cubic component [43].

\section{APPENDIX F: INELASTIC NEUTRON SCATTERING ENERGY SCANS}

Figure 12 uses the experiment performed on the coldneutron time-of-flight spectrometer IN5@ILL to map the phonon spectra in a wide range of energy and momentum at $530[(\mathrm{a}),(\mathrm{b})], 300[(\mathrm{c}),(\mathrm{d})]$, and $150 \mathrm{~K}[(\mathrm{e}),(\mathrm{f})]$. (Further experimental conditions and data integration parameters are detailed in Appendix A.) Figures 12(a), 12(c), and 12(e) follow the transverse polarization centered around the intense (006) Bragg peak in which phonons propagate along [110] and are polarized along [001]. Figures 12(b), 12(d), and 12(f) display the longitudinal polarization, again centered around the (006) Bragg peak, in which phonons propagate along [001].

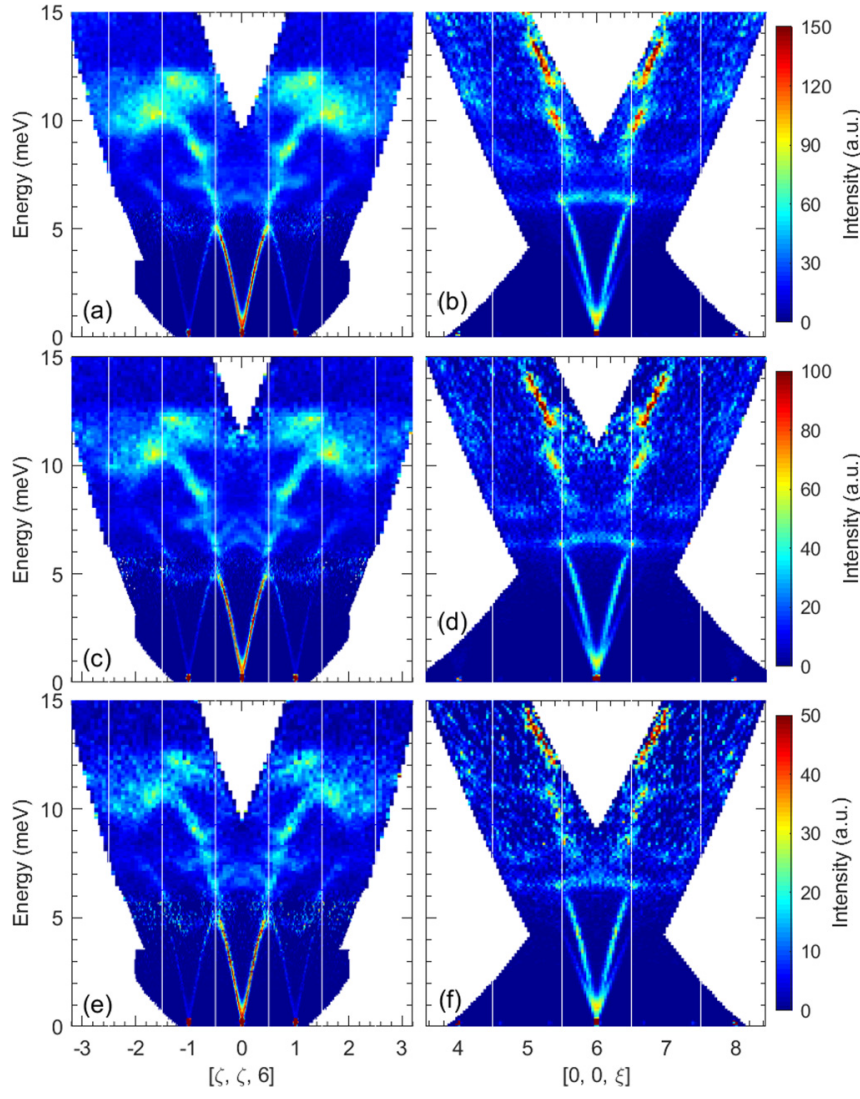

FIG. 12. Two-dimensional inelastic neutron scattering intensity reported as function of the energy transfer and along the [110] [(a), (c), (e)] and [001] [(b), (d), (f)] directions in $\mathrm{Ba}_{7.81} \mathrm{Ge}_{40.67} \mathrm{Au}_{5.33}$, recorded on IN5@ILL. The data have been taken at three temperatures: $530 \mathrm{~K}$ [(a), (b)], $300 \mathrm{~K}$ [(c), (d)], and $150 \mathrm{~K}$ [(e), (f)]. Measurements were centered at the zone center $\Gamma_{006}\left(Q=3.5 \AA^{-1}\right)$, from which the acoustic phonon polarization is longitudinal when the propagation direction is [001] [panels (b), (d), (f)], and transversely polarized [001] when the propagation direction is [110] [panels (a), (c), (e)]. The Brillouin zones are indicated with vertical solid white lines.

Figures 13-15 depict the energy scans taken on IN5@ILL $(\lambda=3.2 \AA)$ around the Bragg peak (006) at $523 \mathrm{~K}, 300 \mathrm{~K}$, and $150 \mathrm{~K}$ respectively. (Experimental conditions and analytical tools also discussed in Appendix A.) These energy scans are like the one-dimensional cuts of the mappings in Fig. 12, and they were used to construct Fig. 3(a). Figures 13(a), 14(a), and 15(a) follow the transverse acoustic phonon dispersion polarized along [001], propagating along [110]. Figures 13(b), 14(b), and 15(b) follow the longitudinal acoustic phonon dispersion polarized along [001], propagating along [001].

Similarly for Fig. 3(b), Figs. 16-18 depict the energy scans taken on IN5@ILL $(\lambda=3.2 \AA)$ around the Bragg peak (222) at $523 \mathrm{~K}, 300 \mathrm{~K}$, and $150 \mathrm{~K}$, respectively. Figures 16(a), 17(a), and 18(a) follow the transverse acoustic phonon dispersion polarized along [111], propagating along [112]. Figures 16(b), 17(b), and 18(b) follow the longitudinal acoustic phonon dispersion polarized along [111], propagating along [111]. Phonons were fitted as Gaussian peaks with a flat background. 

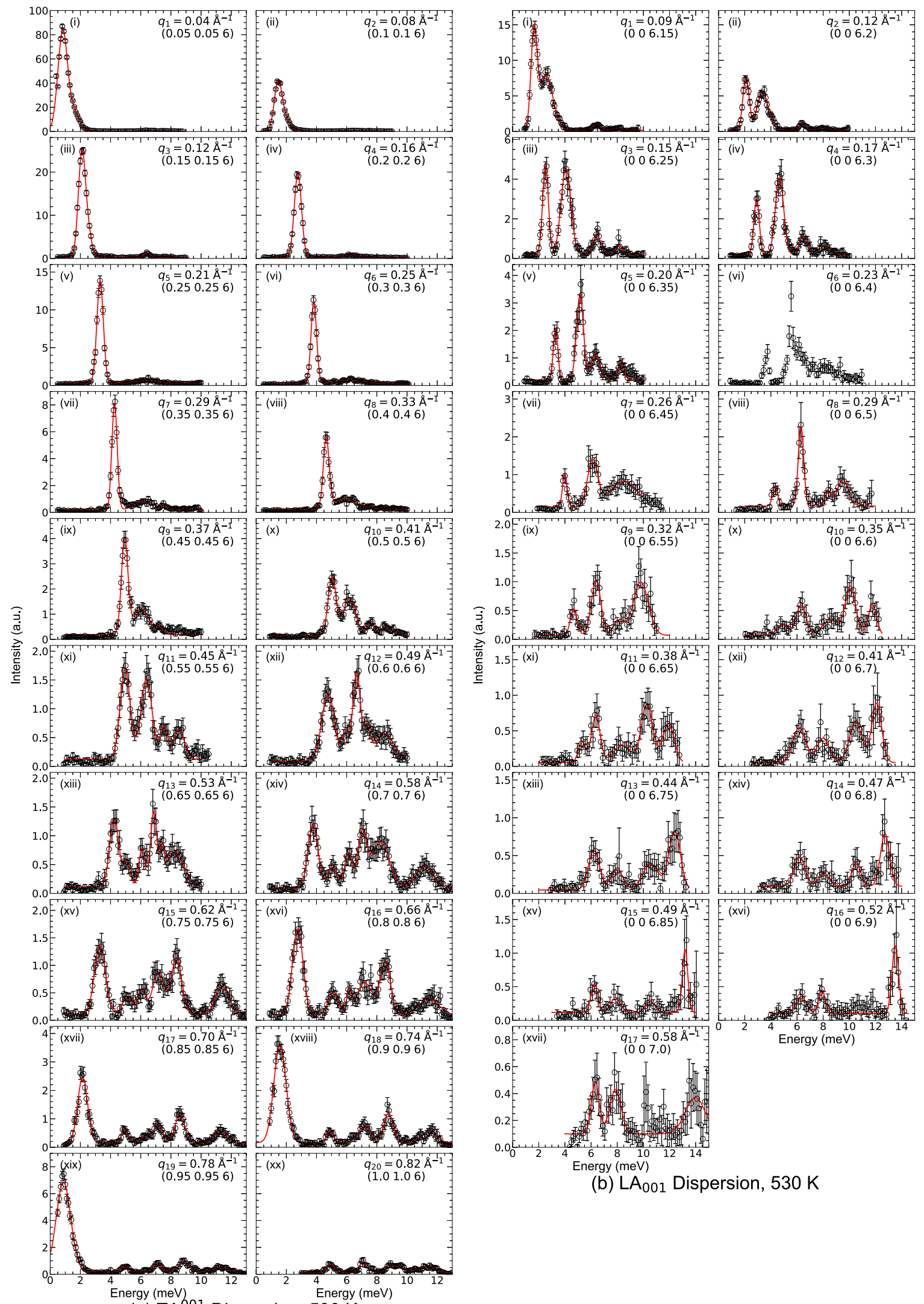

(b) LA 001 Dispersion, $530 \mathrm{~K}$

(a) TA 110 Dispersion, $530 \mathrm{~K}$

FIG. 13. Energy scans taken on IN5@ILL near the (006) Bragg peak with $\lambda=3.2 \AA$ at $530 \mathrm{~K}$. Plots reflect a normalized intensity scale, with data points as empty black circles, and their fit as a solid red line. 


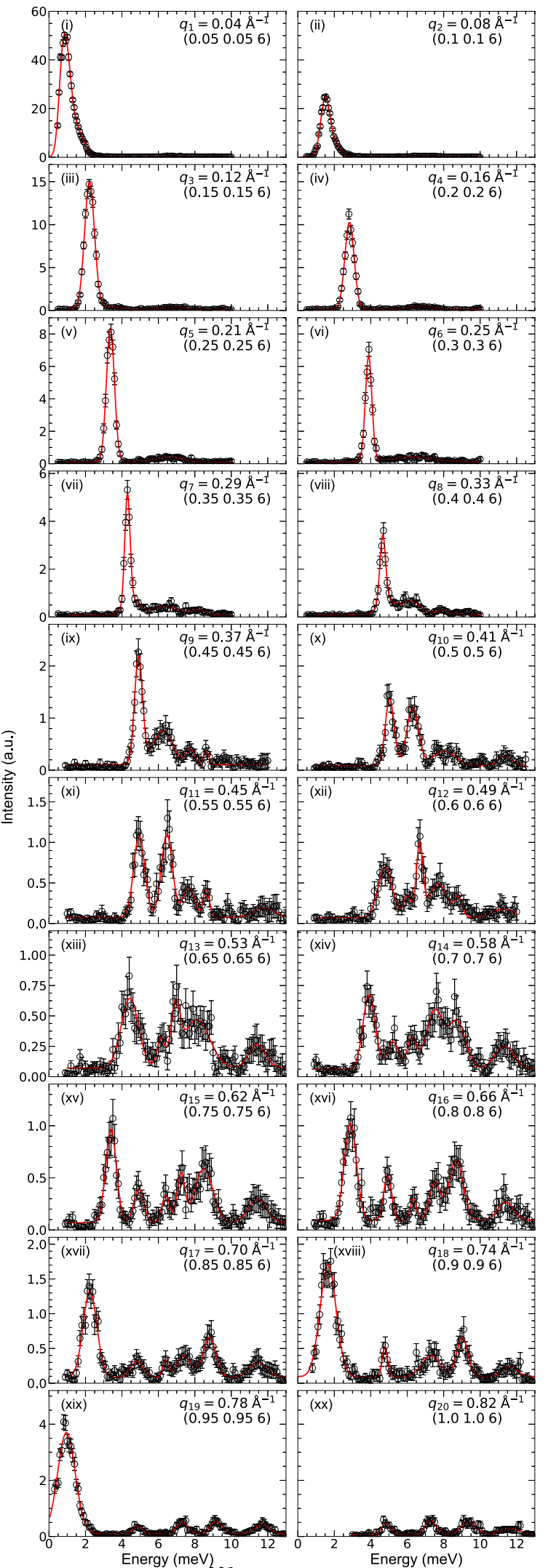

(a) $\mathrm{TA}_{110}^{001}$ Dispersion, $300 \mathrm{~K}$

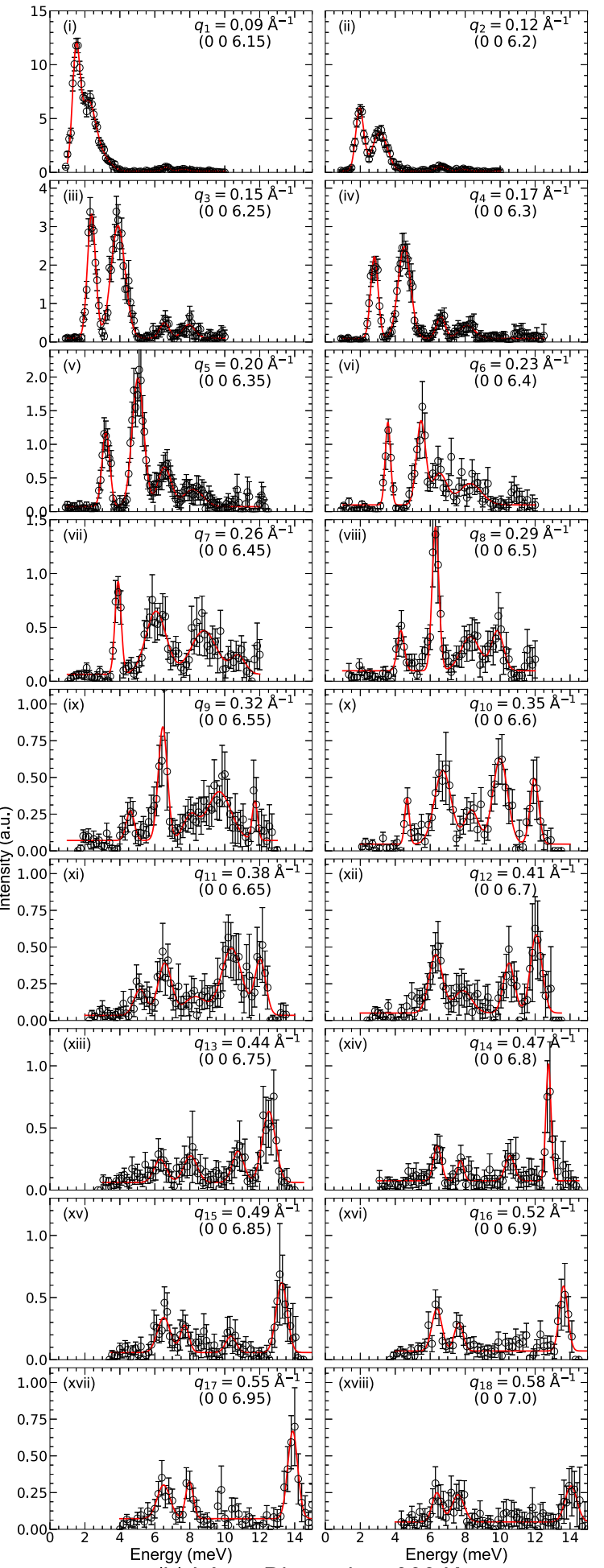

(b) LA $\mathrm{A}_{01}$ Dispersion, $300 \mathrm{~K}$

FIG. 14. Energy scans taken on IN5@ILL near the (006) Bragg peak with $\lambda=3.2 \AA$ at $300 \mathrm{~K}$. Plots reflect a normalized intensity scale, with data points as empty black circles, and their fit as a solid red line. 

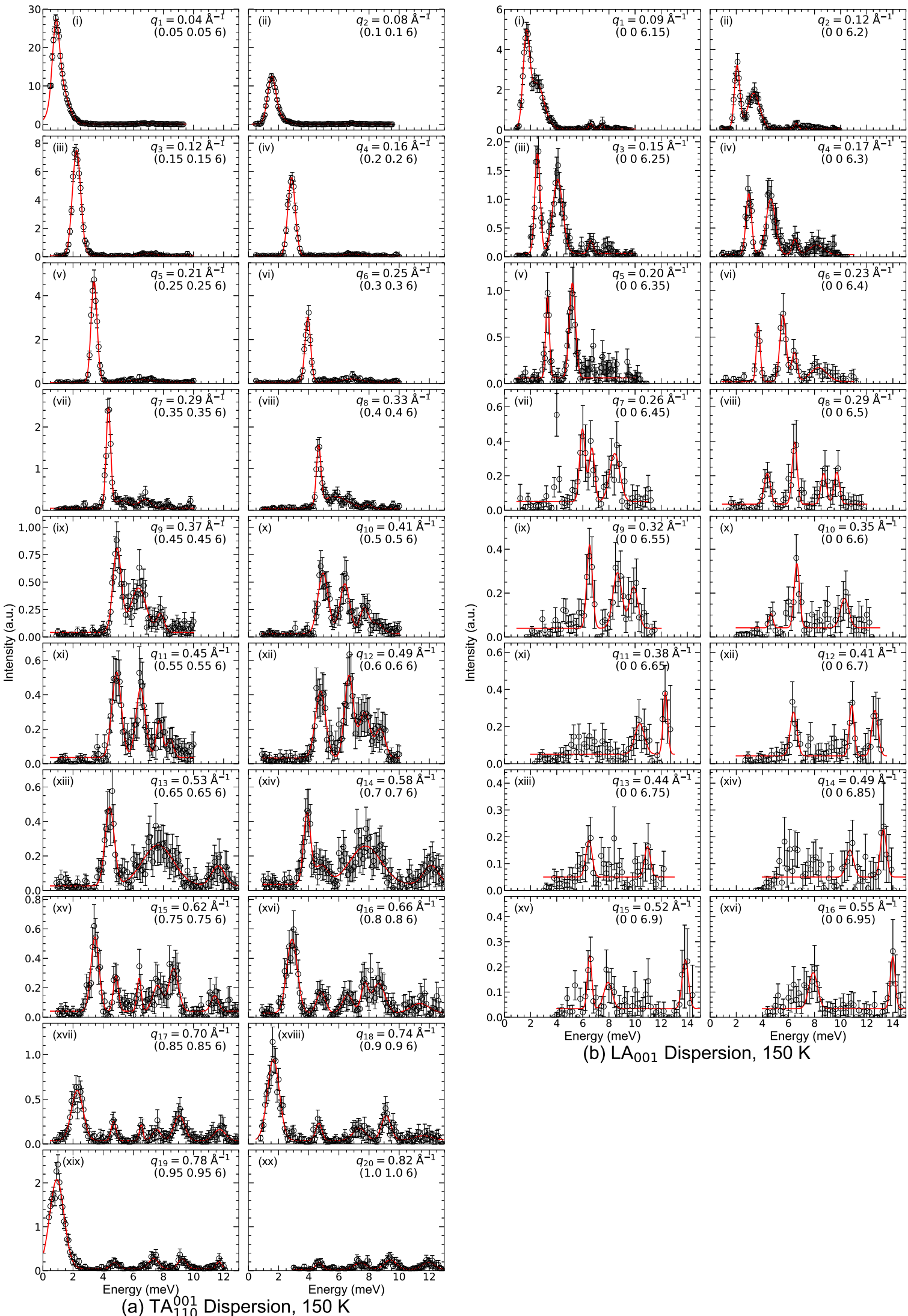

(b) LA $\mathrm{A}_{001}$ Dispersion, $150 \mathrm{~K}$

FIG. 15. Energy scans taken on IN5@ILL near the (006) Bragg peak with $\lambda=3.2 \AA$ at $150 \mathrm{~K}$. Plots reflect a normalized intensity scale, with data points as empty black circles, and the fit as a solid red line. 

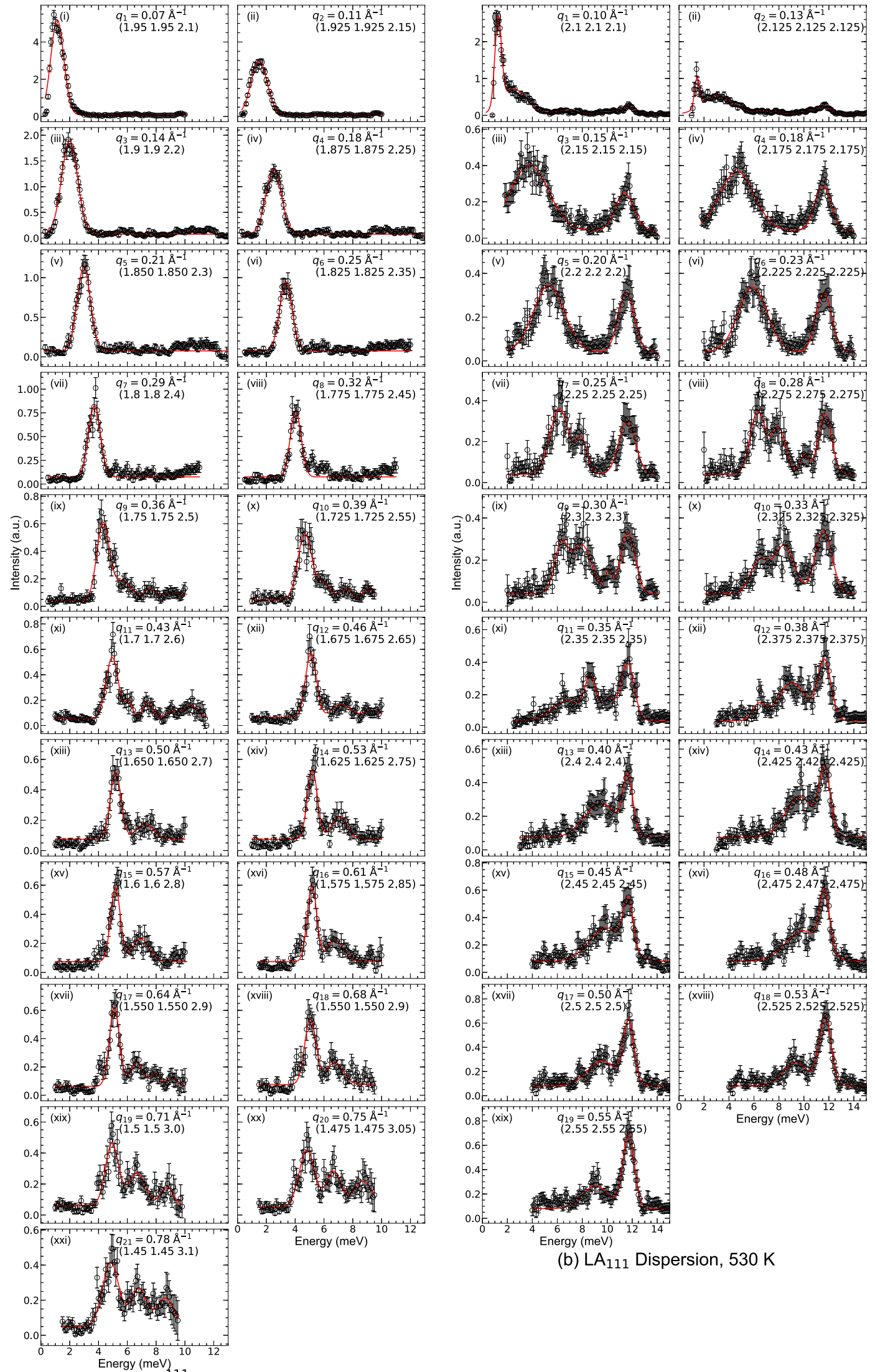

(a) $\mathrm{TA} \frac{111}{112}$ Dispersion, $530 \mathrm{~K}$

FIG. 16. Energy scans taken on IN5@ILL near the (222) Bragg peak with $\lambda=3.2 \AA$ at $530 \mathrm{~K}$. Plots reflect a normalized intensity scale, with data points as empty black circles, and their fit as a solid red line. 

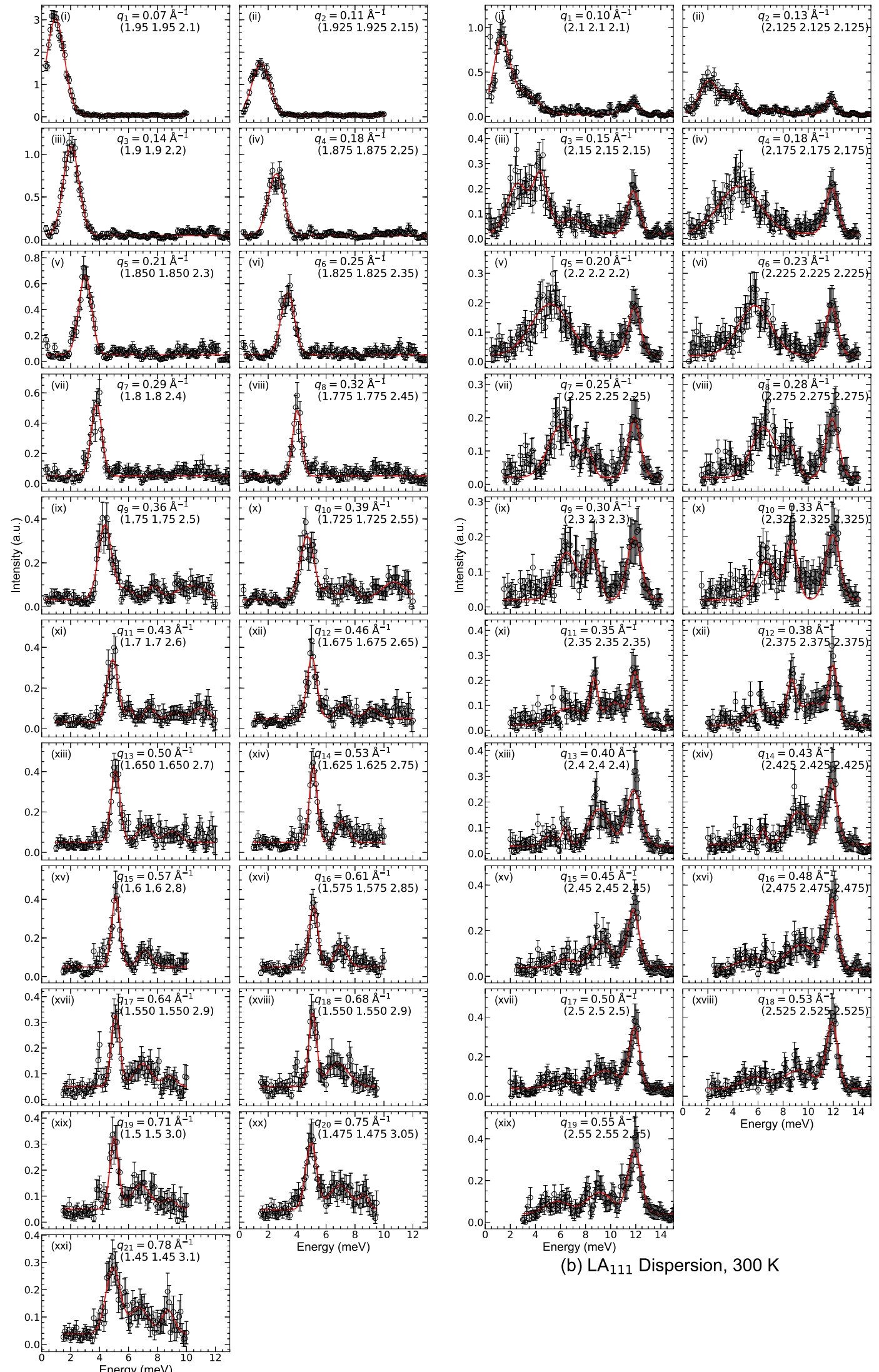

(a) $\mathrm{TA} \frac{111}{112}$ Dispersion, $300 \mathrm{~K}$

FIG. 17. Energy scans taken on IN5@ILL near the (222) Bragg peak with $\lambda=3.2 \AA$ at $300 \mathrm{~K}$. Plots reflect a normalized intensity scale, with data points as empty black circles, and their fit as a solid red line. 

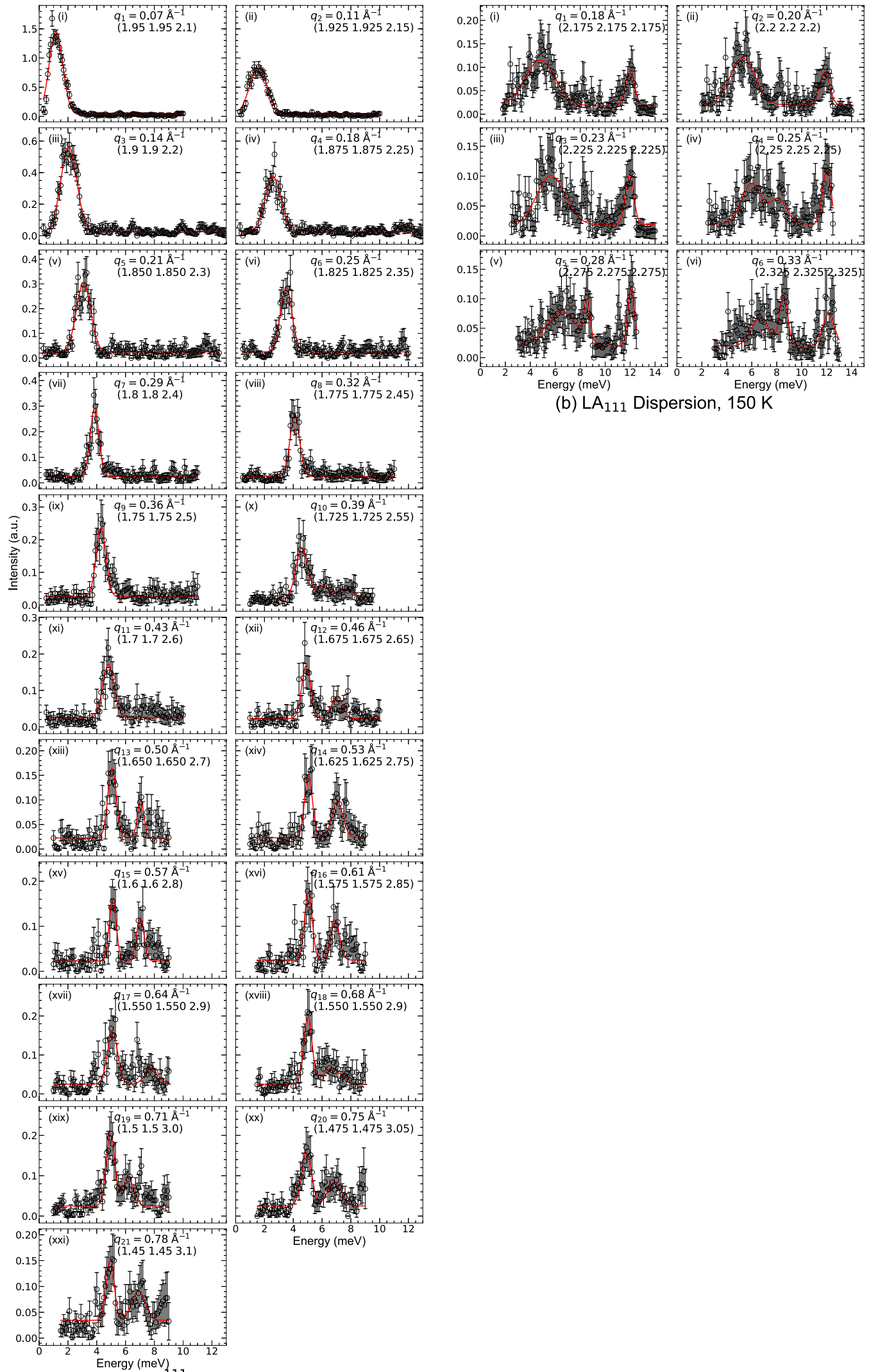

(b) $\mathrm{LA}_{111}$ Dispersion, $150 \mathrm{~K}$

(a) $\mathrm{TA} \frac{111}{112}$ Dispersion, $150 \mathrm{~K}$

FIG. 18. Energy scans taken on IN5@ILL near the (222) Bragg peak with $\lambda=3.2 \AA$ at $150 \mathrm{~K}$. Plots reflect a normalized intensity scale, with data points as empty black circles, and the fit as a solid red line. 
[1] G. J. Snyder and E. S. Toberer, Complex thermoelectric materials, Nat. Mater. 7, 105 (2008).

[2] J. Klarbring, O. Hellman, I. A. Abrikosov, and S. I. Simak, Anharmonicity and Ultra-Low Thermal Conductivity in LeadFree Halide Double Perovskites, Phys. Rev. Lett. 125, 045701 (2020).

[3] J. Even, S. Paofai, P. Bourges, A. Letoublon, S. Cordier, O. Durand, and C. Katan, Carrier scattering processes and low energy phonon spectroscopy in hybrid perovskites crystals, in Physics, Simulation, and Photonic Engineering of Photovoltaic Devices V, edited by A. Freundlich, L. Lombez, and M. Sugiyama (SPIE, 2016), Vol. 9743, pp. 78-85.

[4] Y. Yu, M. Cagnoni, O. Cojocaru-Mirédin, and M. Wuttig, Chalcogenide thermoelectrics empowered by an unconventional bonding mechanism, Adv. Funct. Mater. 30, 1904862 (2019).

[5] H. Yang, J.-y. Yang, C. N. Savory, J. M. Skelton, B. J. Morgan, D. O. Scanlon, and A. Walsh, Highly anisotropic thermal transport in $\mathrm{LiCoO}_{2}$, J. Phys. Chem. Lett. 10, 5552 (2019).

[6] K. Hoang and M. D. Johannes, Defect physics in complex energy materials, J. Phys.: Condens. Matter 30, 293001 (2018).

[7] E. S. Toberer, L. L. Baranowski, and C. Dames, Advances in thermal conductivity, Ann. Rev. Mater. Res. 42, 179 (2012).

[8] E. S. Toberer, A. Zevalkink, and G. J. Snyder, Phonon engineering through crystal chemistry, J. Mater. Chem. 21, 15843 (2011).

[9] A. Verchère, S. Pailhès, S. L. Floch, S. Cottrino, R. Debord, G. Fantozzi, S. Misra, C. Candolfi, B. Lenoir, S. Daniele, and $\mathrm{S}$. Mishra, Optimum in the thermoelectric efficiency of nanostructured $\mathrm{Nb}$-doped $\mathrm{TiO}_{2}$ ceramics: From polarons to $\mathrm{Nb}-\mathrm{Nb}$ dimers, Phys. Chem. Chem. Phys. 22, 13008 (2020).

[10] Y. Grin, Inhomogeneity and anisotropy of chemical bonding and thermoelectric properties of materials, J. Solid State Chem. 274, 329 (2019).

[11] Y. Bouyrie, C. Candolfi, S. Pailhès, M. M. Koza, B. Malaman, A. Dauscher, J. Tobola, O. Boisron, L. Saviot, and B. Lenoir, From crystal to glass-like thermal conductivity in crystalline minerals, Phys. Chem. Chem. Phys. 17, 19751 (2015).

[12] T. Takabatake, K. Suekuni, T. Nakayama, and E. Kaneshita, Phonon-glass electron-crystal thermoelectric clathrates: Experiments and theory, Rev. Mod. Phys. 86, 669 (2014).

[13] P.-F. Lory, S. Pailhès, V. M. Giordano, H. Euchner, H. D. Nguyen, R. Ramlau, H. Borrmann, M. Schmidt, M. Baitinger, M. Ikeda et al., Direct measurement of individual phonon lifetimes in the clathrate compound $\mathrm{Ba}_{7.81} \mathrm{Ge}_{40.67} \mathrm{Au}_{5.33}$, Nat. Commun. 8, 491 (2017).

[14] S. Pailhès, H. Euchner, V. M. Giordano, R. Debord, A. Assy, S. Gomès, A. Bosak, D. Machon, S. Paschen, and M. de Boissieu, Localization of Propagative Phonons in a Perfectly Crystalline Solid, Phys. Rev. Lett. 113, 025506 (2014).

[15] H. Euchner, S. Pailhès, L. T. K. Nguyen, W. Assmus, F. Ritter, A. Haghighirad, Y. Grin, S. Paschen, and M. de Boissieu, Phononic filter effect of rattling phonons in the thermoelectric clathrate $\mathrm{Ba}_{8} \mathrm{Ge}_{40+x} \mathrm{Ni}_{6-x}$, Phys. Rev. B 86, 224303 (2012).

[16] P. Norouzzadeh and C. W. Myles, A first-principles lattice dynamical study of type-I, type-II, and type-VIII silicon clathrates, J. Mater. Sci. 51, 4538 (2016).

[17] H. Euchner, S. Pailhès, V. M. Giordano, and M. de Boissieu, Understanding lattice thermal conductivity in thermoelectric clathrates: A density functional theory study on binary Si-based type-I clathrates, Phys. Rev. B 97, 014304 (2018).

[18] J. Dong, O. F. Sankey, and C. W. Myles, Theoretical Study of The Lattice Thermal Conductivity in Ge Framework Semiconductors, Phys. Rev. Lett. 86, 2361 (2001).

[19] M. S. Ikeda, H. Euchner, X. Yan, P. Tomeš, A. Prokofiev, L. Prochaska, G. Lientschnig, R. Svagera, S. Hartmann, E. Gati, M. Lang, and S. Paschen, Kondo-like phonon scattering in thermoelectric clathrates, Nat. Commun. 10, 887 (2019).

[20] R. Viennois, M. M. Koza, R. Debord, P. Toulemonde, H. Mutka, and $\mathrm{S}$. Pailhès, Anisotropic low-energy vibrational modes as an effect of cage geometry in the binary barium silicon clathrate $\mathrm{Ba}_{24} \mathrm{Si}_{100}$, Phys. Rev. B 101, 224302 (2020).

[21] T. Tadano, Y. Gohda, and S. Tsuneyuki, Impact of Rattlers on Thermal Conductivity of a Thermoelectric Clathrate: A FirstPrinciples Study, Phys. Rev. Lett. 114, 095501 (2015).

[22] M. A. Avila, K. Suekuni, K. Umeo, H. Fukuoka, S. Yamanaka, and T. Takabatake, Glasslike versus crystalline thermal conductivity in carrier-tuned $\mathrm{Ba}_{8} \mathrm{Ga}_{16} \mathrm{X}_{30}$ clathrates $(\mathrm{X}=\mathrm{Ge}, \mathrm{Sn})$, Phys. Rev. B 74, 125109 (2006).

[23] B. C. Sales, B. C. Chakoumakos, R. Jin, J. R. Thompson, and D. Mandrus, Structural, magnetic, thermal, and transport properties of $X_{8} \mathrm{Ga}_{16} \mathrm{Ge}_{30}(X=\mathrm{Eu}, \mathrm{Sr}, \mathrm{Ba})$ single crystals, Phys. Rev. B 63, 245113 (2001).

[24] A. F. May, E. S. Toberer, A. Saramat, and G. J. Snyder, Characterization and analysis of thermoelectric transport in $n$-type $\mathrm{Ba}_{8} \mathrm{Ga}_{16-x} \mathrm{Ge}_{30+x}$, Phys. Rev. B 80, 125205 (2009).

[25] C. Candolfi, M. M. Koza, U. Aydemir, W. Carrillo-Cabrera, Y. Grin, F. Steglich, and M. Baitinger, Vibrational dynamics of the type-I clathrates $A_{8} \mathrm{Sn}_{44} \square_{2}(A=\mathrm{Cs}, \mathrm{Rb}, \mathrm{K})$ from latticedynamics calculations, inelastic neutron scattering, and specific heat measurements, J. Appl. Phys. 127, 145104 (2020).

[26] G. S. Nolas, J. L. Cohn, J. S. Dyck, C. Uher, and J. Yang, Transport properties of polycrystalline type-I Sn clathrates, Phys. Rev. B 65, 165201 (2002).

[27] Y. Liu, Q. Xi, J. Zhou, T. Nakayama, and B. Li, Phonon-glass dynamics in thermoelectric clathrates, Phys. Rev. B 93, 214305 (2016).

[28] Q. Xi, Z. Zhang, J. Chen, J. Zhou, T. Nakayama, and B. Li, Hopping processes explain linear rise in temperature of thermal conductivity in thermoelectric clathrates with off-center guest atoms, Phys. Rev. B 96, 064306 (2017).

[29] S. Johnsen, M. Christensen, B. Thomsen, G. K. H. Madsen, and B. B. Iversen, Barium dynamics in noble-metal clathrates, Phys. Rev. B 82, 184303 (2010).

[30] H. Zhang, H. Borrmann, N. Oeschler, C. Candolfi, W. Schnelle, M. Schmidt, U. Burkhardt, M. Baitinger, J.-T. Zhao, and Y. Grin, Atomic interactions in the p-type clathrate I $\mathrm{Ba}_{8} \mathrm{Au}_{5.3} \mathrm{Ge}_{40.7}$, Inorg. Chem. 50, 1250 (2011).

[31] Z. Ye, J. Y. Cho, M. M. Tessema, J. R. Salvador, R. A. Waldo, J. Yang, H. Wang, W. Cai, M. Kirkham, J. Yang, and W. Zhang, Thermoelectric properties of Au-containing type-I clathrates $\mathrm{Ba}_{8} \mathrm{Au}_{x} \mathrm{Ga}_{16-3 x} \mathrm{Ge}_{30+2 x}$, J. Alloys Compd. 587, 747 (2014).

[32] M. Christensen, N. Lock, J. Overgaard, and B. B. Iversen, Crystal structures of thermoelectric $n$ - and $p$-type $\mathrm{Ba}_{8} \mathrm{Ga}_{16} \mathrm{Ge}_{30}$ studied by single crystal, multitemperature, neutron diffraction, conventional X-ray diffraction and resonant synchrotron X-ray diffraction, J. Am. Chem. Soc. 128, 15657 (2006). 
[33] G. K. H. Madsen, A. Katre, and C. Bera, Calculating the thermal conductivity of the silicon clathrates using the quasiharmonic approximation, Phys. Status Solidi A 213, 802 (2015).

[34] H. Euchner and A. Groß, Predicting accurate phonon spectra: An improved description of lattice dynamics in thermoelectric clathrates based on the SCAN meta-GGA functional, Chem. Mater. 31, 2571 (2019).

[35] P.-F. Lory, V. M. Giordano, P. Gille, H. Euchner, M. Mihalkovič, E. Pellegrini, M. Gonzalez, L.-P. Regnault, P. Bastie, H. Schober et al., Impact of structural complexity and disorder on lattice dynamics and thermal conductivity in the o- $\mathrm{Al}_{13} \mathrm{Co}_{4}$ phase, Phys. Rev. B 102, 024303 (2020).

[36] T. Tadano and S. Tsuneyuki, First-principles lattice dynamics method for strongly anharmonic crystals, J. Phys. Soc. Jpn. 87, 041015 (2018).

[37] I. Errea, M. Calandra, and F. Mauri, Anharmonic free energies and phonon dispersions from the stochastic self-consistent harmonic approximation: Application to platinum and palladium hydrides, Phys. Rev. B 89, 064302 (2014).

[38] T. Lan, C. W. Li, O. Hellman, D. S. Kim, J. A. Muñoz, H. Smith, D. L. Abernathy, and B. Fultz, Phonon quarticity induced by changes in phonon-tracked hybridization during lattice expansion and its stabilization of rutile $\mathrm{TiO}_{2}$, Phys. Rev. B 92, 054304 (2015).

[39] T. Tadano and S. Tsuneyuki, Self-consistent phonon calculations of lattice dynamical properties in cubic $\mathrm{SrTiO}_{3}$ with first-principles anharmonic force constants, Phys. Rev. B 92, 054301 (2015).

[40] W. Sano, T. Koretsune, T. Tadano, R. Akashi, and R. Arita, Effect of van Hove singularities on high- $T_{c}$ superconductivity in $\mathrm{H}_{3} \mathrm{~S}$, Phys. Rev. B 93, 094525 (2016).

[41] Y. Oba, T. Tadano, R. Akashi, and S. Tsuneyuki, First-principles study of phonon anharmonicity and negative thermal expansion in $\mathrm{ScF}_{3}$, Phys. Rev. Materials 3, 033601 (2019).

[42] Y.-N. Wu, W. A. Saidi, J. K. Wuenschell, T. Tadano, P. Ohodnicki, B. Chorpening, and Y. Duan, Anharmonicity explains temperature renormalization effects of the band gap in $\mathrm{SrTiO}_{3}$, J. Phys. Chem. Lett. 11, 2518 (2020).

[43] T. Tadano and S. Tsuneyuki, Quartic Anharmonicity of Rattlers and its Effect on Lattice Thermal Conductivity of Clathrates from First Principles, Phys. Rev. Lett. 120, 105901 (2018).

[44] S. Pailhès, V. M. Giordano, P.-F. Lory, M. D. Boissieu, and H. Euchner, X-rays and neutrons spectroscopy for the investigation of individual phonons properties in crystalline and amorphous solids, in Nanostructured Semiconductors, edited by K. Termentzidis (Jenny Stanford Publishing, New York, 2017), Chap. 19.

[45] G. Kresse and J. Hafner, Ab initio molecular dynamics for liquid metals, Phys. Rev. B 47, 558 (1993).

[46] G. Kresse and J. Furthmüller, Efficient iterative schemes for ab initio total-energy calculations using a plane-wave basis set, Phys. Rev. B 54, 11169 (1996).

[47] G. Kresse and D. Joubert, From ultrasoft pseudopotentials to the projector augmented-wave method, Phys. Rev. B 59, 1758 (1999).

[48] J. Sun, A. Ruzsinszky, and J. P. Perdew, Strongly Constrained and Appropriately Normed Semilocal Density Functional, Phys. Rev. Lett. 115, 036402 (2015).
[49] A. Togo and I. Tanaka, First principles phonon calculations in materials science, Scr. Mater. 108, 1 (2015).

[50] M. Falmbigl, G. Rogl, P. Rogl, M. Kriegisch, H. Müller, E. Bauer, M. Reinecker, and W. Schranz, Thermal expansion of thermoelectric type-I-clathrates, J. Appl. Phys. 108, 043529 (2010).

[51] G. D. Mukherjee, C. Bansal, and A. Chatterjee, Thermal Expansion Study of Ordered and Disordered $\mathrm{Fe}_{3} \mathrm{Al}$ : An Effective Approach for the Determination of Vibrational Entropy, Phys. Rev. Lett. 76, 1876 (1996).

[52] W. Han-Fu, C. Wei-Guo, G. Yan-Jun, and J. Hao, Thermal transport property of $\mathrm{Ge}_{34}$ and d-Ge investigated by molecular dynamics and the Slack's equation, Chinese Phys. B 19, 076501 (2010).

[53] R. L. González-Romero, C. R. Miranda, M. A. Avila, and A. Antonelli, Hosting of $\mathrm{La}^{3+}$ guest ions in type-I Ge clathrates: A first-principles characterization for thermoelectric applications, Comput. Mater. Sci. 122, 46 (2016).

[54] A. Bhattacharya, Deviation from guest dominated glass like lattice dynamics in prototypical ternary $\mathrm{Ba}_{8} \mathrm{Ni}_{x} \mathrm{Ge}_{46-x-y-}$ clathrates, J. Phys.: Condens. Matter 32, 175502 (2020).

[55] A. Khabibullin, T. Huan, G. Nolas, and L. Woods, Cage disorder and gas encapsulation as routes to tailor properties of inorganic clathrates, Acta Mater. 131, 475 (2017).

[56] X. Tang, J. Dong, P. Hutchins, O. Shebanova, J. Gryko, P. Barnes, J. K. Cockroft, M. Vickers, and P. F. McMillan, Thermal properties of $\mathrm{Si}_{136}$ : Theoretical and experimental study of the type-II clathrate polymorph of Si, Phys. Rev. B 74, 014109 (2006).

[57] W. Zhang, Z. Zeng, N. Ge, and Z. Li, Lattice dynamics study of phonon instability and thermal properties of type-I clathrate $\mathrm{K}_{8} \mathrm{Si}_{46}$ under high pressure, Materials 9, 616 (2016).

[58] M. Zebarjadi, E. Keivan, and C. Gang, Thermal conductivity of cage-like structures, ASME/JSME 2011 8th Thermal Engineering Joint Conference, Honolulu, Hawaii, USA (ASME International, 2011).

[59] M. Christensen, A. B. Abrahamsen, N. B. Christensen, F Juranyi, N. H. Andersen, K. Lefmann, J. Andreasson, C. R. H. Bahl, and B. B. Iversen, Avoided crossing of rattler modes in thermoelectric materials, Nat. Mater. 7, 811 (2008).

[60] M. M. Koza, M. R. Johnson, R. Viennois, H. Mutka, L. Girard, and $\mathrm{D}$. Ravot, Breakdown of phonon glass paradigm in La- and Ce-filled $\mathrm{Fe}_{4} \mathrm{Sb}_{12}$ skutterudites, Nat. Mater. 7, 805 (2008).

[61] Y. Takasu, T. Hasegawa, N. Ogita, M. Udagawa, M. A. Avila, K. Suekuni, I. Ishii, T. Suzuki, and T. Takabatake, Dynamical properties of guest ions in the type-I clathrate compounds $X_{8} \mathrm{Ga}_{16} \mathrm{Ge}_{30}(X=\mathrm{Eu}, \mathrm{Sr}, \mathrm{Ba})$ investigated by Raman scattering, Phys. Rev. B 74, 174303 (2006).

[62] Y. Takasu, T. Hasegawa, N. Ogita, M. Udagawa, M. A. Avila, K. Suekuni, and T. Takabatake, Off-Center Rattling and Anisotropic Expansion of Type-I Clathrates Studied by Raman Scattering, Phys. Rev. Lett. 100, 165503 (2008).

[63] Y. Takasu, T. Hasegawa, N. Ogita, M. Udagawa, M. A. Avila, K. Suekuni, and T. Takabatake, Off-center rattling and cage vibration of the carrier-tuned type-I clathrate $\mathrm{Ba}_{8} \mathrm{Ga}_{16} \mathrm{Ge}_{30}$ studied by Raman scattering, Phys. Rev. B 82, 134302 (2010).

[64] M. M. Koza, M. R. Johnson, H. Mutka, M. Rotter, N. Nasir, A. Grytsiv, and P. Rogl, Vibrational dynamics of the type-I clathrate $\mathrm{Ba}_{8} \mathrm{Zn}_{x} \mathrm{Ge}_{46-x-y} \square_{y}(x=0,2,4,6,8)$, Phys. Rev. B 82, 214301 (2010). 
[65] Data for this paper are available at https://doi.ill.fr/10.5291/ ILL-DATA.7-01-494 and https://doi.ill.fr/10.5291/ILL-DATA. 7-01-449.

[66] www.ecmetac.eu.

[67] G. Shirane, S. M. Shapiro, and J. M. Tranquada, Neutron Scattering with a Triple-Axis Spectrometer (Cambridge University Press, Cambridge, 2015).

[68] O. Arnold, J. Bilheux, J. Borreguero, A. Buts, S. Campbell, L. Chapon, M. Doucet, N. Draper, R. F. Leal, M. Gigg et al., Mantid-Data analysis and visualization package for neutron scattering and $\mu$ SR experiments, Nucl. Instrum. Meth. A 764, 156 (2014)

[69] R. Ewings, A. Buts, M. Le, J. van Duijn, I. Bustinduy, and T. Perring, Horace: Software for the analysis of data from single crystal spectroscopy experiments at time-offlight neutron instruments, Nucl. Instrum. Meth. A 834, 132 (2016).

[70] G. Ehlers, A. A. Podlesnyak, J. L. Niedziela, E. B. Iverson, and P. E. Sokol, The new cold neutron chopper spectrometer at the Spallation Neutron Source: Design and performance, Rev. Sci. Instrum. 82, 085108 (2011).

[71] W. Reichardt, MUPHOCOR, A Fortran program to determine the phonon density of states from neutron scattering experiments, Report No. 13.03.01P06L, Institut für Nukleare Festkorper Physik, Kernforschung Szentrum, Karlsruhe GMBH, Germany.

[72] D. Richard, M. Ferrand, and G. J. Kearley, Analysis and visualisation of neutron-scattering data, J. Neutron Res. 4, 33 (1996).
[73] M. T. Rekveldt, T. Keller, and R. Golub, Larmor precession, a technique for high-sensitivity neutron diffraction, Europhys. Lett. 54, 342 (2001).

[74] T. Keller, M. Rekveldt, and K. Habicht, Neutron Larmor diffraction measurement of the lattice-spacing spread of pyrolytic graphite, Appl. Phys. A: Mater. 74, s127 (2002).

[75] M. Ono, Y. Waku, K. Habicht, and T. Keller, Neutron larmor diffraction measurement of strain in a ductile composite $\mathrm{Al}_{2} \mathrm{O}_{3} / \mathrm{Y}_{3} \mathrm{Al}_{5} \mathrm{O}_{12}$ (YAG), Appl. Phys. A: Mater. 74, s73 (2002).

[76] T. Keller, P. Fabrykiewicz, R. Przeniosło, I. Sosnowska, and B. Keimer, Neutron Larmor diffraction on powder samples, J. Appl. Crystallogr. 53, 88 (2020).

[77] F. I. Fedorov, Theory of Elastic Waves in Crystals (Springer US, New York, 1968).

[78] A. Authier and A. Zarembowitch, in International Tables for Crystallography, edited by A. Authier, Vol. D (Springer, Dordrecht, 2006), Chap. 1.3, pp. 72-98.

[79] F. Mouhat and F.-X. Coudert, Necessary and sufficient elastic stability conditions in various crystal systems, Phys. Rev. B 90 , 224104 (2014).

[80] N. L. Okamoto, T. Nakano, K. Tanaka, and H. Inui, Mechanical and thermal properties of single crystals of the type-I clathrate compounds $\mathrm{Ba}_{8} \mathrm{Ga}_{16} \mathrm{Ge}_{30}$ and $\mathrm{Sr}_{8} \mathrm{Ga}_{16} \mathrm{Ge}_{30}$, J. Appl. Phys. 104, 013529 (2008).

[81] I. Zerec, V. Keppens, M. A. McGuire, D. Mandrus, B. C. Sales, and P. Thalmeier, Four-Well Tunneling States and Elastic Response of Clathrates, Phys. Rev. Lett. 92, 185502 (2004).

[82] F. H. Stillinger, Energy Landscapes, Inherent Structures, and Condensed-Matter Phenomena (Princeton University Press, Princeton, 2015). 